\title{
The Analysis and Prediction of the 8-9 May 2007 Oklahoma Tornadic Mesoscale Convective System by Assimilating WSR-88D and CASA Radar Data Using 3DVAR
}

\author{
Alexander D. Schenkman, Ming Xue, and Alan Shapiro \\ Center for Analysis and Prediction of Storms, and School of Meteorology, \\ University of Oklahoma, Norman, Oklahoma \\ KEITH BREWSTER AND JiDONG GAO \\ Center for Analysis and Prediction of Storms, University of Oklahoma, Norman, Oklahoma
}

(Manuscript received 6 January 2010, in final form 11 August 2010)

\begin{abstract}
The Advanced Regional Prediction System (ARPS) model is employed to perform high-resolution numerical simulations of a mesoscale convective system and associated cyclonic line-end vortex (LEV) that spawned several tornadoes in central Oklahoma on 8-9 May 2007. The simulation uses a $1000 \mathrm{~km} \times 1000 \mathrm{~km}$ domain with $2-\mathrm{km}$ horizontal grid spacing. The ARPS three-dimensional variational data assimilation (3DVAR) is used to assimilate a variety of data types. All experiments assimilate routine surface and upperair observations as well as wind profiler and Oklahoma Mesonet data over a 1-h assimilation window. A subset of experiments assimilates radar data. Cloud and hydrometeor fields as well as in-cloud temperature are adjusted based on radar reflectivity data through the ARPS complex cloud analysis procedure. Radar data are assimilated from the Weather Surveillance Radar-1988 Doppler (WSR-88D) network as well as from the Engineering Research Center for Collaborative and Adaptive Sensing of the Atmosphere (CASA) network of four X-band Doppler radars. Three-hour forecasts are launched at the end of the assimilation window. The structure and evolution of the forecast MCS and LEV are markedly better throughout the forecast period in experiments in which radar data are assimilated. The assimilation of CASA radar data in addition to WSR-88D data increases the structural detail of the modeled squall line and MCS at the end of the assimilation window, which appears to yield a slightly better forecast track of the LEV.
\end{abstract}

\section{Introduction}

With increases in computing power, the explicit prediction of convective storms is becoming a reality. As computing power continues to increase, it will likely be possible to produce these explicit convective storm forecasts in real time. Examples of these real-time forecasts have already been produced as part of the Hazardous Weather Test bed spring experiments (Kong et al. 2009; Xue et al. 2009). These forecasts are the first efforts in fulfilling the National Weather Service's "warn on forecast" vision (Stensrud et al. 2009).

Corresponding author address: Alex Schenkman, School of Meteorology, University of Oklahoma, 120 David L. Boren Blvd., Norman, OK 73072.

E-mail: alex3238@ou.edu
One of the major challenges with storm-resolving numerical weather prediction (NWP) is the ability to produce an accurate initial model condition. Because NWP is a highly nonlinear initial value problem, the accuracy of the prediction is fundamentally limited by the accuracy of the initial analyzed state. The goal of this study is to produce (in a way that is practical for real-time implementation) an accurate analysis and forecast of a tornadic mesoscale convective system (MCS) that occurred in southwest and central Oklahoma on 8-9 May 2007. Toward that end, we examine the utility of the Advanced Regional Prediction System (ARPS; Xue et al. 2000, 2001, 2003) three-dimensional variational data assimilation (3DVAR; e.g., Gao et al. 2004) package for assimilating data from a variety of sources, including radar reflectivity and radial velocity data from the operational Weather Surveillance Radar-1988 Doppler (WSR-88D) 
radar network and the National Science Foundation (NSF) Engineering Research Center for Collaborative Adaptive Sensing of the Atmosphere (CASA) Integrated Project One (IP1) X-band radar network.

The assimilation of reflectivity and velocity data from Doppler radars is vital to predicting ongoing convection because radar is the only observation platform with the temporal and spatial resolution sufficient to resolve convective storms. Numerous studies (e.g., Sun and Crook 1998; Weygandt et al. 2002a, b; Xue et al. 2003; Xiao et al. 2005; Dawson and Xue 2006; Hu et al. 2006a, b) have shown reasonable success in simulating and forecasting convective storms when radar data are assimilated.

A number of methods exist for the assimilation of radar data. Sun et al. (1991) and Sun and Crook (1997, 1998) have shown that four-dimensional variational analysis (4DVAR), a procedure in which a numerical model is fit to observations over a time window, is an effective way to assimilate radar data because it takes advantage of the high temporal resolution of Doppler radar as well as prognostic (and dynamical) constraints. The goal of 4DVAR data assimilation is to determine an initial model state that will produce output parameters that match observations as closely as possible over a data assimilation window. 4DVAR assimilation of radar data, however, has so far been limited to relatively simple model configurations, usually only with warmrain microphysics (Sun 2005). Strong nonlinearity with model physics, including ice microphysics, often causes difficulties with the minimization convergence in 4DVAR.

Ensemble Kalman filter (EnKF) is another advanced method for assimilating radar data (Snyder and Zhang 2003; Zhang et al. 2004; Tong and Xue 2005; Xue et al. 2006). By using a Monte Carlo sampling technique with ensembles of numerical model forecasts, EnKF determines flow-dependent error correlation statistics (Evensen 1994) so that unobserved state variables of the atmosphere can be "retrieved" from limited radar observations (Tong and Xue 2005). Caya et al. (2005) showed through simulated-data experiments that EnKF and 4DVAR produce analyses of generally similar quality, with a model containing warm-rain microphysics. Unfortunately, both methods are rather expensive computationally, especially when used at the convection-resolving scale, and/or for real-time applications.

A computationally cheaper alternative to $4 \mathrm{DVAR}$ and EnKF is to analyze radar data via a 3DVAR analysis procedure. The ARPS 3DVAR system (Xue et al. 2003; Gao et al. 2004; Hu et al. 2006b) is capable of analyzing radar radial velocity data along with conventional observations. The 3DVAR formulation is less theoretically optimal than 4DVAR because it lacks a time dimension and thus cannot incorporate prognostic constraints. However, a time dimension can be brought in by running experiments with high-frequency intermittent analysis cycles to make better use of data distributed in time ( $\mathrm{Hu}$ et al. 2006a,b). Hu and Xue (2007) showed that the assimilation window length and assimilation frequency must be carefully considered. The ARPS 3DVAR is described in greater detail in section 2.

Our study focuses on an MCS and an associated line end vortex (LEV; e.g., Weisman 1993) that passed through southwest and central Oklahoma on 8-9 May 2007. The MCS and LEV were observed by the NSF Engineering Research Center for CASA's (McLaughlin et al. 2009) Integrated Project One (IP1) radar network.

The CASA IP1 network consists of four X-band dualpolarization radars located in southwest Oklahoma. One of the goals of the CASA project is to develop and test small-wavelength, short range (maximum range of $30 \mathrm{~km}$ ), low-cost, and low-powered radars in high-density networks. These networks are designed to sample the lower atmosphere more effectively than long-range less-dense networks, such as the operational WSR-88D radars.

The CASA-IP1 network was installed between two WSR-88Ds radars in Oklahoma: KTLX at Oklahoma City and KFDR in Frederick. This location was chosen because it is climatologically upstream of the Oklahoma City metropolitan area, and neither WSR-88D covers the lowest kilometer of the atmosphere in this region (Xue et al. 2006). Thus, CASA radars can serve to fill the low-level data gap left by the WSR-88D radars. Even though the CASA radars have a relatively broad beamwidth $\left(1.8^{\circ}\right.$ vs the $0.89^{\circ}$ of WSR-88D), their range gate spacing of $100 \mathrm{~m}$ (vs $250 \mathrm{~m}$ for WSR-88D), adaptive sampling capabilities, and the short baseline of the CASA radars allow for much higher spatial and temporal resolution than typically available with WSR-88D radars. Such finescale resolution is paramount for observing small-scale features such as tornadoes (Brotzge et al. 2010).

In this study, we perform a set of experiments aimed at producing a quality forecast of the 9 May 2007 LEV and MCS, using an efficient 3DVAR analysis procedure that is practical for real-time implementations. At the same time, we examine, for the first time, the impact of assimilating data from the new experimental X-band radars from the CASA IP-1 network described above. This paper is organized as follows. In section 2, the 9 May 2007 LEV case is described. Section 3 summarizes the ARPS assimilation and prediction systems. Section 4 describes the numerical experiments and verification method. In section 5, we discuss the analysis and forecast results. A summary and conclusions are given in section 6 . 


\section{8-9 May 2007 LEV}

The LEV of 8-9 May 2007 produced several weak tornadoes that struck parts of southwest and central Oklahoma on 9 May 2007. The LEV formed out of a large convective system that developed around 1200 UTC 8 May 2007 in far eastern New Mexico in an area of upslope flow and moisture advection. The thunderstorm complex grew in areal coverage and extended from southwest Oklahoma south to near Del Rio, Texas, at 0000 UTC 9 May 2007. The Texas portion of the complex began to dissipate shortly after 0100 UTC while new supercell-like development in southwest Oklahoma allowed the line to persist until about 0730 UTC.

At approximately 2200 UTC 8 May 2007 a cyclonic LEV developed in the northern portion of the MCS in the vicinity of Wichita Falls, Texas. The development of the LEV occurred as the MCS merged with a supercell to its northeast. This evolution is similar to vortex formation mechanism shown in the strong shear simulations of Weisman and Davis (1998) as well as to an observational study of the evolution of a supercell-bow echo interaction presented in Wolf (1998).

The LEV strengthened and contracted while moving north-northeast into southwest Oklahoma. A second supercell in Comanche County, Oklahoma (Fig. 1a), was absorbed by the MCS at about 0245 UTC (Figs. 1b,c). Once again, the interaction with a supercell led to the intensification of the LEV. This intensification was evident in both the radar reflectivity pattern and 10-m wind observations (Fig. 1d) from the Oklahoma Mesonet. The LEV reached its maximum intensity between 0400 and 0500 UTC (Figs. 1e,f). At 0510 UTC radar and Oklahoma Mesonet data indicate the LEV passed within $1 \mathrm{~km}$ of the El Reno Mesonet site. Wind observations from the El Reno Mesonet site indicate a wind shift from the east at $10 \mathrm{~m} \mathrm{~s}^{-1}$ at $0500 \mathrm{UTC}$ to the southwest at $15 \mathrm{~m} \mathrm{~s}^{-1}$ by 0520 UTC (Fig. 2). This suggests a well-defined surface circulation with about $25 \mathrm{~m} \mathrm{~s}^{-1}$ of shear across the LEV. The LEV began to weaken after 0530 UTC and a circulation was no longer evident in radar imagery by 0700 UTC. The overall evolution of the MCS and LEV closely resembles the conceptual model presented in Fujita (1978). Throughout much of its life cycle the MCS also conforms well to the conceptual model of the asymmetric convective system shown in Houze et al. (1989).

\section{ARPS model parameters and 3DVAR}

\section{a. ARPS model and grid configuration}

The ARPS is used as the prediction model in this study. ARPS is a general-purpose three-dimensional, nonhydrostatic, compressible atmospheric model (Xue et al. 2000, 2001, 2003). The common configurations for all experiments include fourth-order advection in both the horizontal and vertical; a rigid top boundary condition combined with a wave absorbing layer, fourth-order computational mixing, 1.5-order TKE-based subgridscale turbulent mixing scheme, and PBL parameterization; and Lin et al. (1983) three-ice microphysics with the rain intercept parameter set to $8.0 \times 10^{5} \mathrm{~m}^{-4}$ according to Snook and Xue (2008). Surface fluxes are calculated using surface temperature and moisture content predicted by the soil model, and radiative processes are calculated from the National Aeronautics and Space Administration (NASA) Goddard Space Flight Center (GSFC) long- and shortwave radiation package. More details on the physics package can be found in Xue et al. (2001).

The data assimilation and forecast experiments are conducted on a $2-\mathrm{km}$ horizontal-resolution grid that is $1000 \mathrm{~km} \times 1000 \mathrm{~km}$ in size and is centered at $34.80^{\circ} \mathrm{N}$, $98.00^{\circ} \mathrm{W}$. It covers all of Oklahoma, the northern half of Texas, southern Kansas, and far southeastern Colorado (Fig. 3a, the quantitative verification domain considered in section 4 is also highlighted). A Lambert conformal map projection is used. The grid is stretched in the vertical, with 43 levels and a minimum vertical grid spacing of $100 \mathrm{~m}$ near the surface. Grid stretching is calculated according to a cubic function of height. Figure $3 \mathrm{~b}$ shows the location of several counties in Oklahoma that are referenced in this study.

Fields from the 0000 UTC NCEP 12-km resolution North American model (NAM) analysis were interpolated to the 2-km ARPS grid to provide the initial analysis background. Lateral boundary conditions were updated at 3-h intervals from the 0000 UTC NAM forecast.

Terrain data were derived from the U.S. Geographical Survey 3-s dataset. A multilayer soil model in the ARPS that is similar to the Oregon State University Noah land surface model (Chen and Dudhia 2001) is used, with five vertical soil levels.

\section{b. ARPS 3DVAR and data sources}

The ARPS 3DVAR minimizes a cost function that includes the background, observation, and mass conservation constraint terms. Following Gao et al. (2004), this cost function may be written as

$$
\begin{aligned}
J(x)= & \frac{1}{2}\left(\mathbf{x}-\mathbf{x}_{b}\right)^{\mathrm{T}} \mathbf{B}^{-1}\left(\mathbf{x}-\mathbf{x}_{b}\right) \\
& +\frac{1}{2}\left[H(\mathbf{x})-\mathbf{y}_{o}\right]^{\mathrm{T}} \mathbf{R}^{-1}\left[H(\mathbf{x})-\mathbf{y}_{o}\right]+J_{c},
\end{aligned}
$$



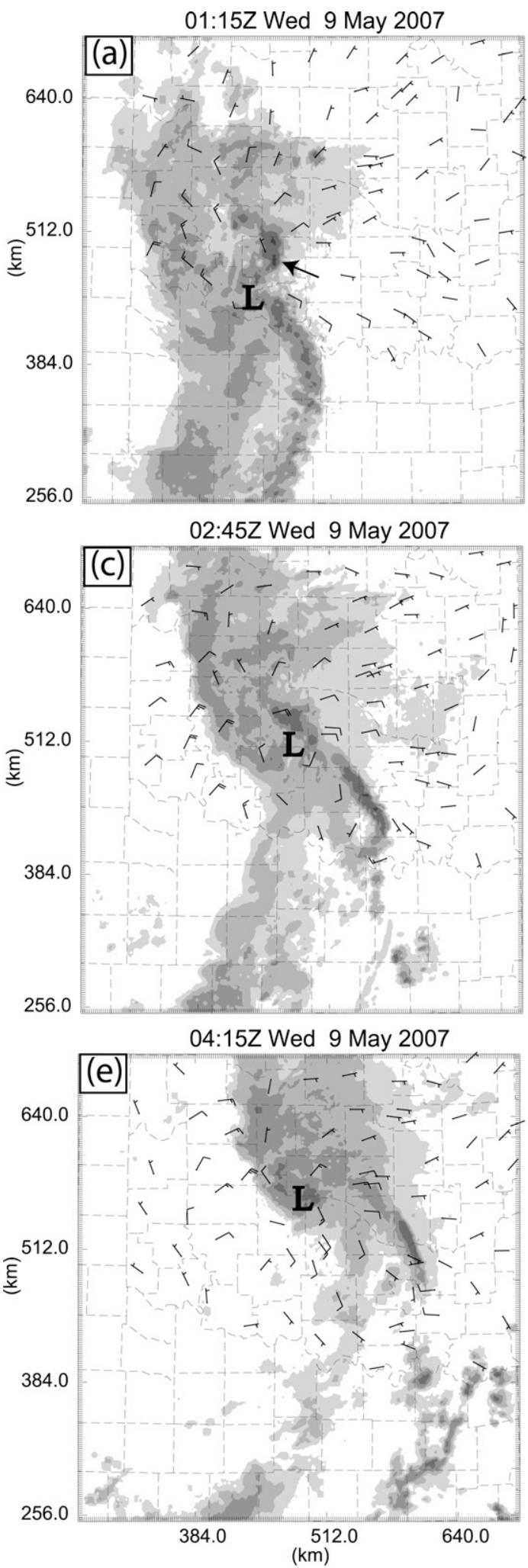
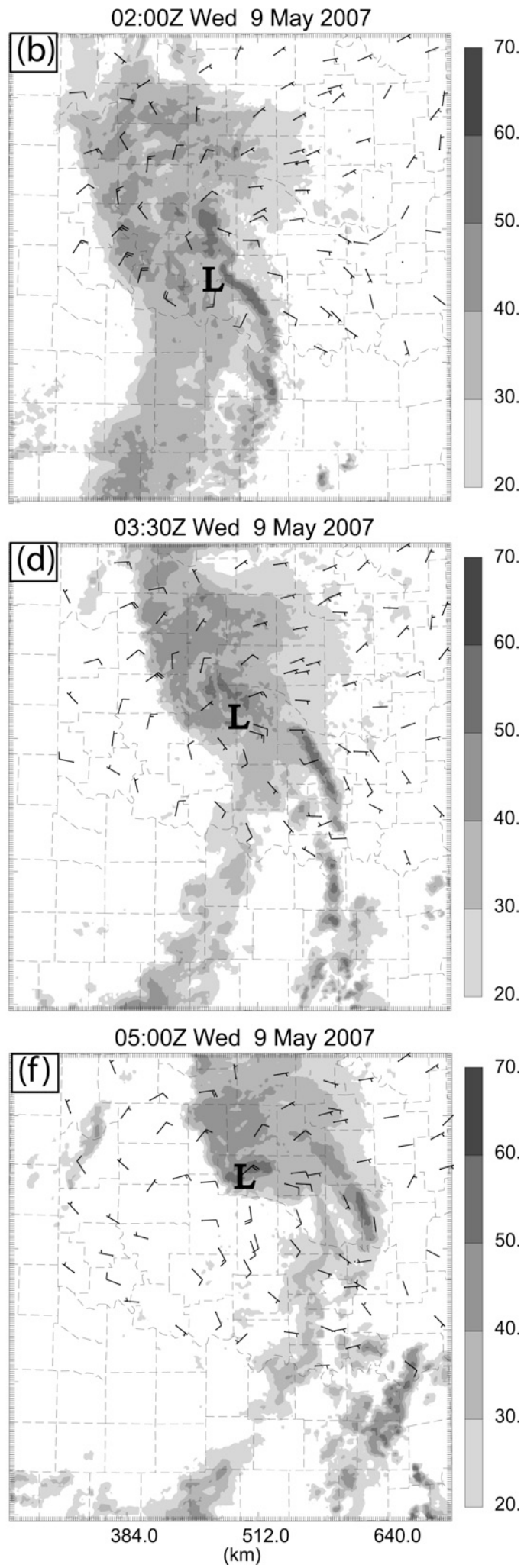

FIG. 1. Mosaic of observed composite radar reflectivity (in dBZ, shaded) with 10-m Oklahoma Mesonet wind observations ( $\mathrm{m} \mathrm{s}^{-1}$ ) overlaid at (a) 0115, (b) 0200, (c) 0245, (d) 0330, (e) 0415, and (f) 0500 UTC 9 May 2007. The "L" corresponds to the location of the LEV center as deduced from KTLX and KFDR radial velocity observations. The arrow in (a) points to the supercell referenced in the text. 


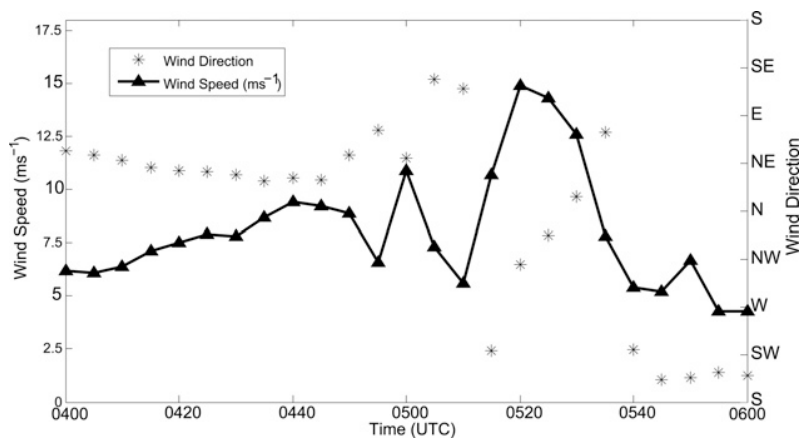

FIG. 2. Five-minute $10-\mathrm{m}$ wind direction and speed $\left(\mathrm{m} \mathrm{s}^{-1}\right)$ observations from 0400 through 0600 UTC 9 May 2007, from the El Reno Mesonet site.

where the first term measures the departure of the analysis vector $\mathbf{x}$ from the background vector $\mathbf{x}_{\mathrm{b}}$, weighted by the inverse of the background error covariance matrix $\mathbf{B}$, and the second term measures the departure of $\mathbf{x}$, projected into observation space by $H$, from the observation vector $\mathbf{y}_{o}$. The third term in (1), $J_{c}$, is a penalty term consisting of a weak mass divergence constraint imposed on the analyzed wind field to help constrain wind components that are perpendicular to the radar beam [see Gao et al. (2004) and Hu et al. (2006b) for more details]. The analysis vector $\mathbf{x}$ includes the three wind components $(u, v$, and $w)$, potential temperature $\theta$, pressure $p$, and water vapor mixing ratio $q_{v}$. Hydrometeors are not analyzed variationally. Because no appropriate balance condition between analysis variables exists at the convective scales modeled in this study, the cross correlations between variables are not included in $\mathbf{B}$. In addition, flowdependent spatial covariance structures are generally not available in a 3DVAR framework. In the ARPS 3DVAR, the spatial covariance of background error is assumed Gaussian, spatially homogeneous, and isotropic. It is modeled using a one-dimensional recursive filter applied successively in each of the three directions. The interested reader is referred to Gao et al. (2004) for more details on the use of recursive filters and the practical implementation of the cost function minimization (e.g., the transformation of $\mathbf{x}$ to a preconditioned control variable and the minimization algorithm).

As is common practice, observation errors are assumed to be uncorrelated, hence the observation error covariance matrix $\mathbf{R}$ is diagonal. The observation error variances are specified according to estimated errors for the various observational platforms (Table 1). Given the lack of reliable statistics on the scales of the background error correlation, and the practical issues of analyzing observations with very different network density (e.g., mesonet versus radar), multiple analysis passes are used to analyze different data types with different (recursive)
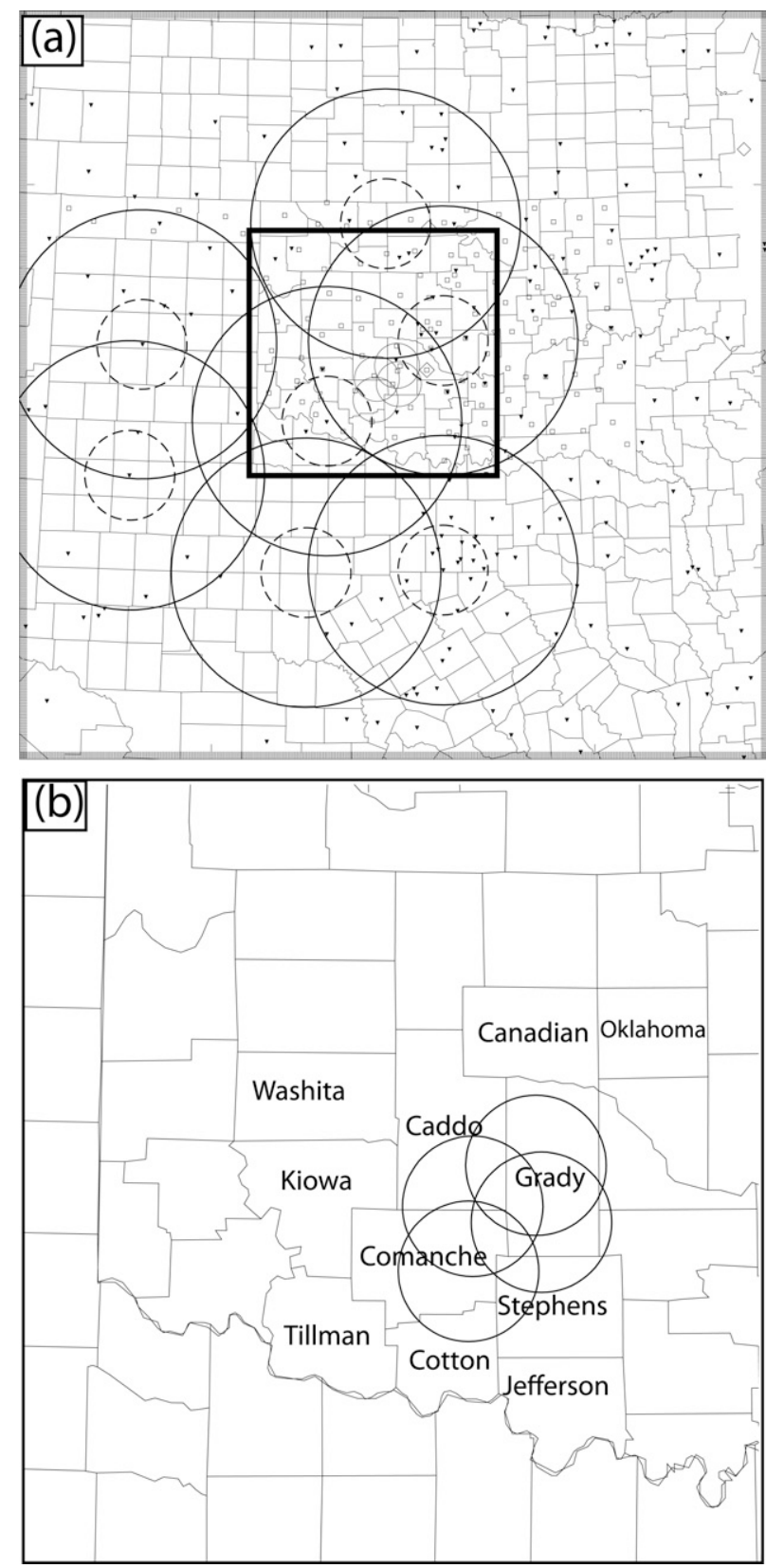

FIG. 3. (a) Map of the $1000 \mathrm{~km} \times 1000 \mathrm{~km}$ computational domain. The interior black rectangle denotes the domain over which quantitative verification statistics are calculated. The small squares represent locations of assimilated Oklahoma Mesonet sites. The filled triangles are locations of assimilated ASOS sites, and the diamonds are locations of assimilated profilers. The dotted circles represent the $60-\mathrm{km}$-range rings of the assimilated WSR-88D, while the solid large circles are the $180-\mathrm{km}$-range rings. (b) Selected county names in southwest and central Oklahoma. In both (a) and (b), the small solid circles represent the maximum range $(30 \mathrm{~km})$ of the CASA IP1 radars. 
TABLE 1. Specified observation error covariance values for all assimilated data types. If a range of values is given, it refers to the specified error variance from near surface to the highest-altitude observation.

\begin{tabular}{lccccr}
\hline \multicolumn{1}{c}{ Data source } & $u\left(\mathrm{~m} \mathrm{~s}^{-1}\right)$ & $v\left(\mathrm{~m} \mathrm{~s}^{-1}\right)$ & Pressure $(\mathrm{hPa})$ & Temperature $(\mathrm{K})$ & Relative humidity $(\%)$ \\
\hline Raobs & $2.0-3.5$ & $2.0-3.5$ & $0.6-0.4$ & $1.0-2.0$ & $5-15$ \\
Profiler & $2.0-3.5$ & $2.0-3.5$ & $0.6-0.4$ & $1.0-2.0$ & 1.11 \\
MDCRS & 1.5 & 1.5 & 1.22 & 0.60 & 1.11 \\
ASOS & 1.0 & 1.0 & 2.00 & 5.0 \\
Oklahoma Mesonet & 1.5 & 1.5 & 2.00 & & 5.0 \\
& Radial velocity $\left(\mathrm{m} \mathrm{s}^{-1}\right)$ & & & \\
WSR-88D & 2.0 & & & \\
CASA IP-1 & 2.5 & & & \\
\hline
\end{tabular}

filter decorrelation scales in order to account for the variations in the observation spacing among different data sources. Here we define the filter decorrelation scale as the radius at which the weight given to the observation in the recursive filter is $e$-folded. The choice of the filter decorrelation scales is guided by the density of observational networks whose data are analyzed within each pass. Such a procedure was used in earlier studies based on the ARPS 3DVAR (e.g., Hu et al. 2006a,b), and a similar procedure using multiple passes with variable spatial correlation scales is used in the ARPS Data Analysis System (ADAS; Brewster 1996) based on a successive correction method (e.g., Xue and Martin 2006).

In this study, we use three analysis passes with decreasing filter decorrelation scale on each pass. Upperair data from raobs and wind profilers are analyzed in the first analysis pass because these data have the coarsest horizontal resolutions and the data tend to represent the synoptic scales. The horizontal filter decorrelation scale for these data is set to $200 \mathrm{~km}$. Surface observations from ASOS and the Oklahoma Mesonet (Brock et al. 1995) as well as Meteorological Data Collection and Reporting System (MDCRS) aircraft observations are analyzed in the second pass. The horizontal decorrelation scale for these data is set to $75 \mathrm{~km}$. The vertical decorrelation scale is set to four grid intervals in both the first and second passes.

Radar radial velocity data are used in the third analysis pass. Level-II data are used from six WSR-88D radars: Twin Lakes (KTLX), Vance (KVNX), Dyess (KDYX),
Amarillo (KAMA), Dallas-Fort Worth (KDFW), and Lubbock (KLBB), Texas. Level-III reflectivity data are used from KFDR because level-II data from that radar were not available from the National Climatic Data Center (NCDC). In experiments using CASA data, data from all four CASA radars are used. For the highresolution radar data, the horizontal decorrelation scale is reduced to $4 \mathrm{~km}$. The vertical scale is set to two grid intervals. Table 2 summarizes the analysis parameters for the various data sources.

\section{c. Radar data processing and quality control}

Automated quality control is performed to unfold and remove noise from the radar data. Anomalous propagation is removed by rejecting radial velocity and reflectivity data where the spectrum width is greater than two-thirds of the Nyquist velocity, as well as where large vertical gradients of reflectivity are present. Radial velocity is also rejected if reflectivity is less than $-5 \mathrm{dBZ}$. A lower threshold of $15 \mathrm{~dB} Z$ is imposed on reflectivity, reflectivity below this threshold (and not equal to a specified missing value) is set to 0 . The quality-controlled radar data are then mapped from radar coordinates onto model grid points using a local least squares fit. Additional details of the radar remapping and quality-control algorithms can be found in Brewster et al. (2005).

Because data from both the CASA and WSR-88D radars are mapped to the model grid resolution and a 4-km horizontal decorrelation radius is used in the 3DVAR assimilation, the differences in radar resolution

TABLE 2. List of assimilated data types and 3DVAR analysis properties.

\begin{tabular}{lcccc}
\hline \hline \multicolumn{1}{c}{ Data source } & Analysis pass & $\begin{array}{c}\text { Horizontal decorrelation } \\
\text { radius }(\mathrm{km})\end{array}$ & $\begin{array}{c}\text { Vertical decorrelation radius } \\
\text { (No. of grid points) }\end{array}$ & Assimilation frequency \\
\hline Raobs & 1 & 200 & 4 & 0000 UTC only \\
Profiler & 1 & 200 & 4 & Hourly \\
ASOS & 2 & 75 & 4 & Hourly \\
Oklahoma Mesonet & 2 & 75 & 4 & Hourly \\
MDCRS & 2 & 75 & 2 & Hourly \\
WSR-88D Vr & 3 & 4 & 2 & 5 min \\
CASA IP-1 Vr & 3 & 4 & & 5 min \\
\hline
\end{tabular}


between the two platforms are likely smoothed out. To take advantage of the higher-resolution sampling capabilities of the CASA radars (mentioned in the introduction) we would need to use a very high-resolution model grid $(\sim 100 \mathrm{~m})$; however, this is not computationally feasible in a quasi-operational setting at this time. Even though the potential resolution benefits are degraded by radar preprocessing and the 3DVAR analysis, the assimilation of CASA data still should impact the analyses via CASA's ability to observe regions beneath the lowest sweep of the nearest WSR-88D radars.

The original ARPS radar data preprocessor, 88d2arps, was designed for the standard WSR-88D $360^{\circ}$ volumescanning coverage patterns. CASA radars collect data dynamically and adaptively, typically as a combination of full $360^{\circ}$ scans and smaller sector scans. The scan strategy is described in more detail in McLaughlin et al. (2009). To apply 88d2arps to the CASA radar data, we build a pseudovolume out of a number of sector scans collected over $1 \mathrm{~min}$ (the shortest time interval at which the scanning strategy can be changed). This data processing procedure was described in Brewster et al. (2007).

\section{d. Cloud analysis}

Variational analysis of reflectivity in 3DVAR is difficult because of the lack of physical constraints to properly attribute to the contributions of multiple hydrometeor species to the reflectivity. A direct link between reflectivity and temperature (or moisture) does not exist and therefore reflectivity cannot directly update these variables within a 3DVAR framework without reliable flowdependent cross covariance. For these reasons, we employ a complex cloud analysis procedure to assimilate reflectivity data that has proven effective in past studies (Xue et al. 2003; Hu et al. 2006a; Zhao and Xue 2009). The cloud analysis is performed as an additional step after the 3DVAR analysis, and utilizes the 3DVAR analysis to provide background information, including that of vertical velocity to determine if convection is present in a particular column.

Within the cloud analysis, the remapped reflectivity data are used to estimate hydrometeor fields via the Kessler (1969) reflectivity equation for rainwater and Rogers and Yau (1989) equations for hail and snow. The in-cloud temperature and moisture fields are estimated by assuming a modified moist-adiabatic ascent that accounts for entrainment as presented by $\mathrm{Hu}$ et al. (2006a). Because radar-observed reflectivity is generally much more reliable than the model prediction, the hydrometeor fields estimated from observations replace the background field everywhere observations are available. This helps to remove spurious precipitation in the forecast background. Where reflectivity observations are
TABLE 3. List of experiments and the radar datasets they assimilate.

\begin{tabular}{lcccc}
\hline \hline Expt name & $\begin{array}{c}\text { WSR-88D } \\
Z \text { data }\end{array}$ & $\begin{array}{c}\text { WSR-88D } \\
\text { Vr data }\end{array}$ & $\begin{array}{c}\text { CASA } \\
Z \text { data }\end{array}$ & $\begin{array}{c}\text { CASA } \\
\text { Vr data }\end{array}$ \\
\hline NORAD & No & No & No & No \\
88DONLY & Yes & Yes & No & No \\
CASAZ & Yes & Yes & Yes & No \\
CASAVr & Yes & Yes & No & Yes \\
CASAVrZ & Yes & Yes & Yes & Yes \\
\hline
\end{tabular}

not available, the background field is retained. More information on the cloud analysis can be found in Zhang et al. (1998), Zhang (1999), Brewster (2002), and Hu et al. (2006a).

The cloud analysis was originally developed to alleviate the spinup problem for forecasts beginning from a coarse-resolution analysis. Repeated application of the original cloud analysis in the high-frequency assimilation cycles of our study, however, led to unrealistic warming in the middle troposphere. To mitigate this problem, the cloud analysis was modified so that the cloud water and water vapor mixing ratios were only adjusted during the first application of the analysis. In subsequent analyses, only the hydrometeor mixing ratios (rain, snow, and hail) and in-cloud temperatures were adjusted.

\section{Experiment design and verification method}

\section{a. Experiments}

For our assimilation-forecast experiments, a 1-h "spinup" forecast is first performed. This spinup forecast starts from a 3DVAR analysis at 0000 UTC 9 May 2007 using all conventional observations (i.e., all observations types other than radar observations in Table 1) and the NAM analysis as the background. Fields at the end of this 1-h forecast serve as the background for the first analysis of the high-frequency, 5-min interval, intermittent analysis cycles, which run from 0100 to 0200 UTC 9 May. All experiments assimilate conventional observations at the start (0100 UTC) and end (0200 UTC) times of this high-frequency assimilation window. Forecasts are then launched from the final analysis at 0200 UTC and run up to 0500 UTC 9 May.

The experiments differ based upon what (if any) radar data are assimilated during the 5-min intermittent analysis cycles. The combinations of radar data used in the experiments are summarized in Table 3. With the exception of one experiment, which assimilates no radar data (NORAD), all experiments assimilate both radial velocity $\mathrm{Vr}$ and reflectivity $Z$ from the WSR-88D radars listed in section $2 \mathrm{~b}$. To examine the impact of CASA 
data, additional experiments assimilate WSR-88D data plus CASA Vr data only (CASAVr), CASA $Z$ data only (CASAZ), and both CASA Vr and $Z$ data (CASAVrZ). Experiment 88DONLY uses radar data from the WSR88 D network only.

\section{b. Verification}

Experiment results are analyzed via qualitative comparison with one another as well as quantitative verification against observations. The latter is performed by calculating the reflectivity correlation coefficient $r_{c}$ and number of reflectivity $N_{R}$ as defined in Aksoy et al. (2010, hereafter ADS10). Our calculations differ from ADS10 in that we calculate $r_{c}$ and $N_{R}$ against a mosaic of observed radar reflectivity in model space rather than in observation space. The mosaic of observed reflectivity uses data from KTLX, KVNX, KFWS, and KFDR. Given these differences, reflectivity correlation coefficient (adapted from ADS10) is defined as

$$
r_{c}=\frac{\sum_{i=1}^{M}\left(Z_{m}-\left\langle Z_{m}\right\rangle\right)\left(Z_{o}-\left\langle Z_{o}\right\rangle\right)}{\left[\sum_{i=1}^{M}\left(Z_{m}-\left\langle Z_{m}\right\rangle\right)^{2}\left(Z_{o}-\left\langle Z_{o}\right\rangle\right)^{2}\right]^{1 / 2}},
$$

where $Z_{m}$ is model reflectivity, $Z_{o}$ is observed reflectivity interpolated into model space, brackets denote the mean over the verification domain, and the summation is over all points in the verification domain with observed reflectivity of greater than or equal to $15 \mathrm{~dB} Z$. The $15-\mathrm{dB} Z$ threshold was also used in ADS10 and is used to limit verification to the main areas of convective and stratiform precipitation. To further focus verification on the LEV and related convection we limit our verification domain to a $330 \mathrm{~km} \times 320 \mathrm{~km}$ area highlighted in Fig. 3a. This area was chosen because it encompasses the LEV and most of the associated convection throughout the forecast period. In addition, in order to minimize contamination of statistics from nonmeteorological scatterers and the melting layer (bright band), we calculate $r_{c}$ and $N_{R}$ at grid level 7 (about $1100 \mathrm{~m} \mathrm{AGL)} \mathrm{which} \mathrm{is} \mathrm{high} \mathrm{enough} \mathrm{to} \mathrm{re-}$ duce the nonmeteorological (e.g., biological scatterers) and low enough to avoid brightband contamination. This differs from ADS10 in which all levels are included in the calculations.

Reflectivity correlation coefficient was chosen for verification because it is less sensitive to location errors than the Gilbert skill score (Schaefer 1990), a statistic more commonly known as the equitable threat score (ETS). The statistic $N_{R}$ is a measure of the total number of grid points within the verification domain with a reflectivity value exceeding the $40-\mathrm{dB} Z$ threshold. Because reflectivity of $40 \mathrm{~dB} Z$ is generally only present in strong convection (ADS10), the evolution of $N_{R}$, combined with qualitative verification, is a reasonable measure of the evolution of the amount/intensity of active convection within the verification domain.

We also note here that verification via reflectivity is used in the absence of direct observations of the kinematic field with sufficient density and coverage to verify forecast positions of gust fronts and other storm features. In particular, multi-Doppler wind synthesis from the CASA network did not cover enough of the storm for verification purposes, while single-Doppler radial velocity measurements were sensitive to spatial errors in the forecast, which made verification using these data difficult (see the discussion at the end of section 5).

\section{Results}

In this section, we present results from the experiments described in section 3a. Particular attention is given to contrasting the forecasts from the various experiments as well as comparing with the observed evolution of the LEV. Before discussing the forecast results, we first describe in detail the final analysis (0200 UTC) from each experiment. This allows us to isolate the direct impact of the different data types assimilated on the analyzed fields. Our discussion of the forecast period will show how these differences in the analyses impact the resulting forecast evolution of the LEV and associated convection between the various experiments.

\section{a. Assimilation results}

The 0200 UTC analyses from all experiments as well as the observed reflectivity are shown in Fig. 4. Observed radar reflectivity shows a large MCS over much of Oklahoma and north Texas at this time (Fig. 4a). The analysis from NORAD (Fig. 4b) is unable to capture the MCS and instead features an elongated north-northeastsouth-southeast band of light precipitation over much of far southwestern Oklahoma. To the east of this precipitation band, an area of convective cells is present. In contrast, the reflectivity field associated with the MCS is accurately represented in the 0200 UTC analysis from the radar-assimilating (RA) experiments (Figs. 4c-f). This result is expected, as these experiments assimilate the observed reflectivity through the cloud analysis.

Near the surface, the NORAD analysis indicates a large cold pool with a gradual wind shift at the leading edge (Fig. 5a). A weak cyclonic vortex is present on the leading edge of the cold pool. The cold pool and vortex are only present in the lowest few grid levels. This indicates that they are likely the result of assimilating 

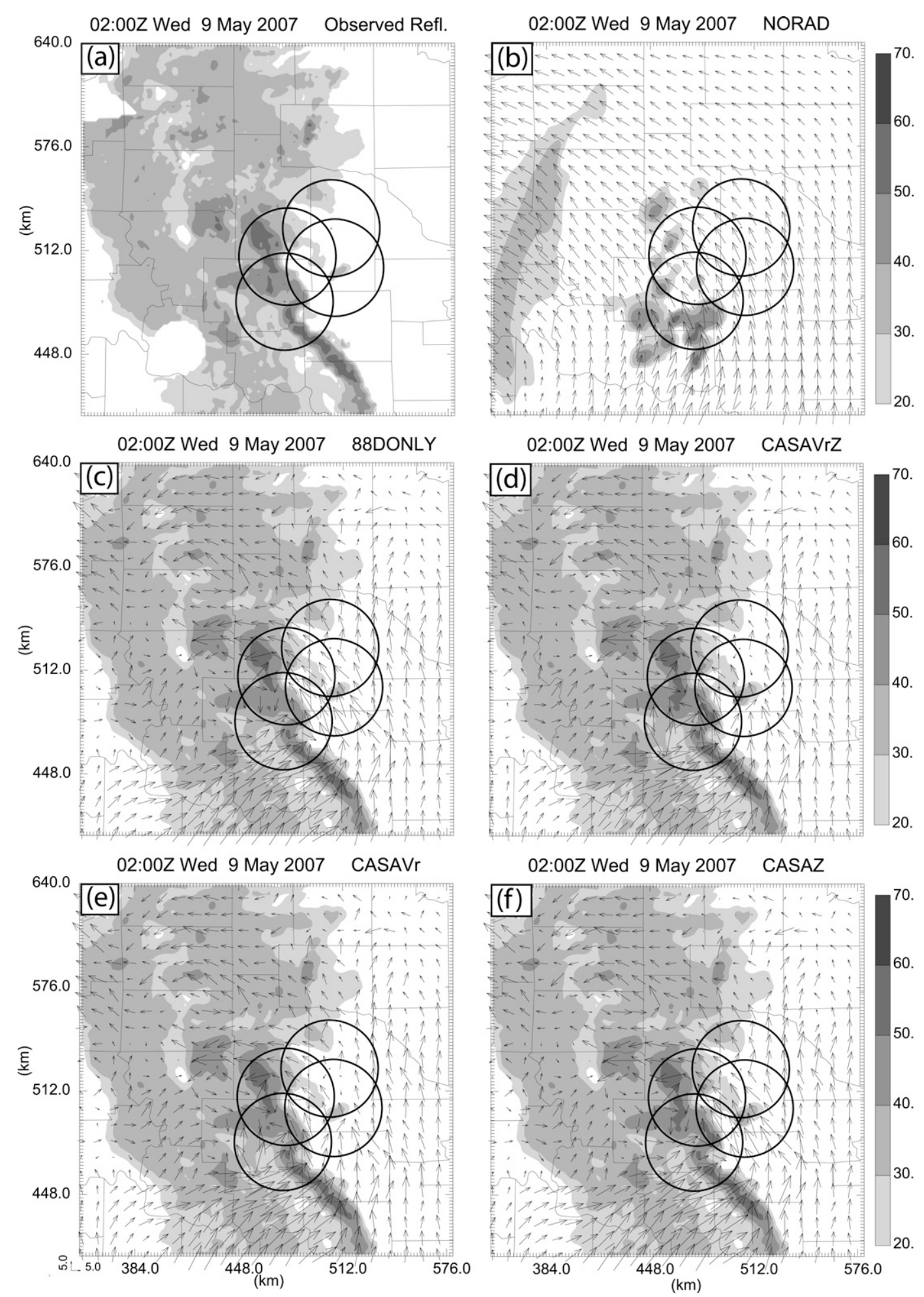

FIG. 4. (a) Observed reflectivity factor (shaded, dBZ) from the radar mosaic at 0200 UTC 9 May 2007. Analyzed reflectivity field (shaded, dBZ) and horizontal wind vectors $\left(\mathrm{m} \mathrm{s}^{-1}\right)$ at $0200 \mathrm{UTC}$ from (b) NORAD, (c) 88DONLY, (d) CASAVrZ, (e) CASAVr, and (f) CASAZ. Black circles are as in Fig. 3. All fields are plotted at grid level 7 (about $1100 \mathrm{~m}$ AGL). 

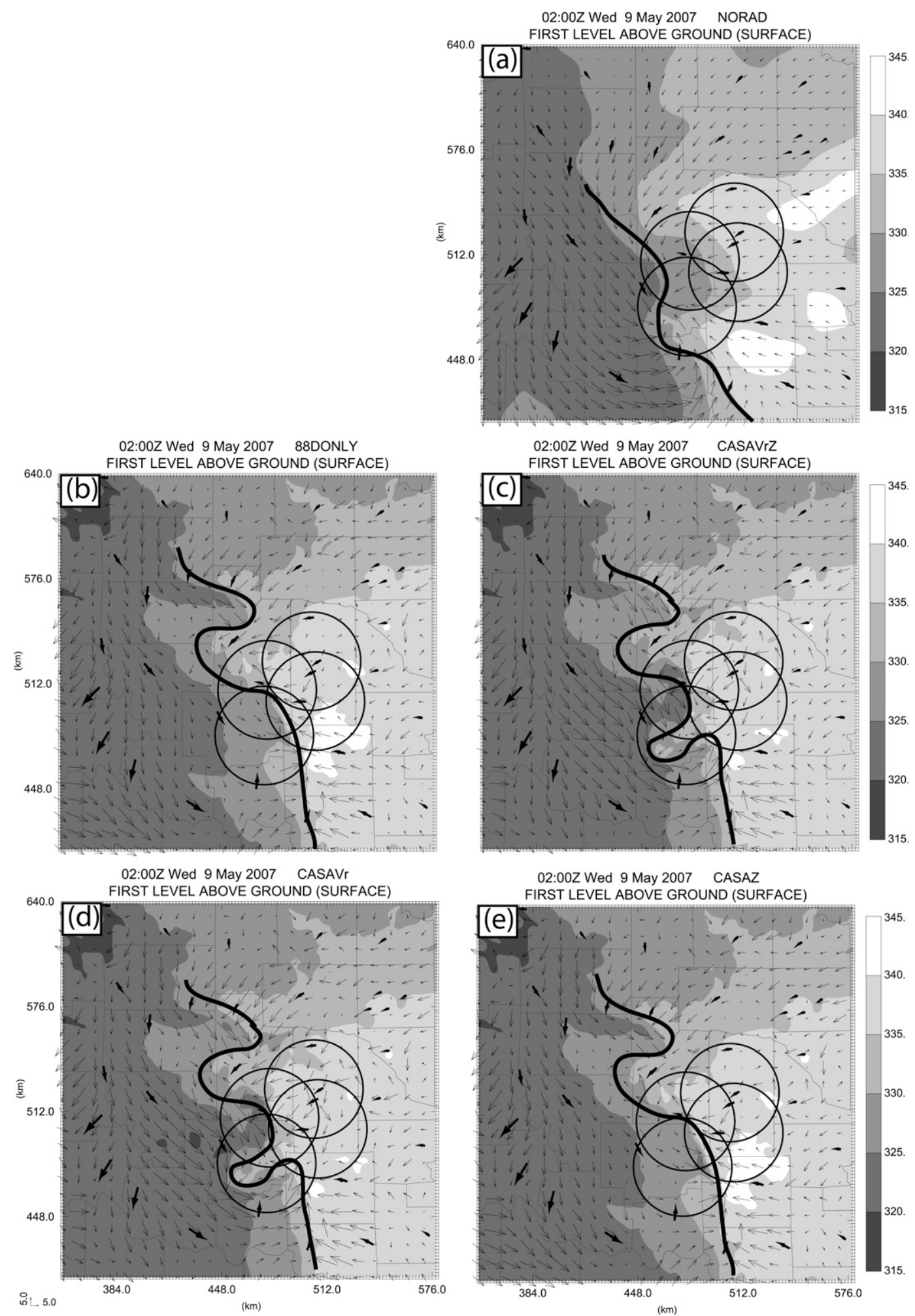

FIG. 5. The 0200 UTC 9 May 2007 near-surface analyses from (a) NORAD, (b) 88DONLY, (c) CASAVrZ, (d) CASAVr, and (e) CASAZ. Equivalent potential temperature is shaded in 5-K intervals, and vectors are horizontal winds $\left(\mathrm{m} \mathrm{s}^{-1}\right)$. Thick black vectors are Oklahoma Mesonet observations. The thick black line marks the approximate location of the gust front. 

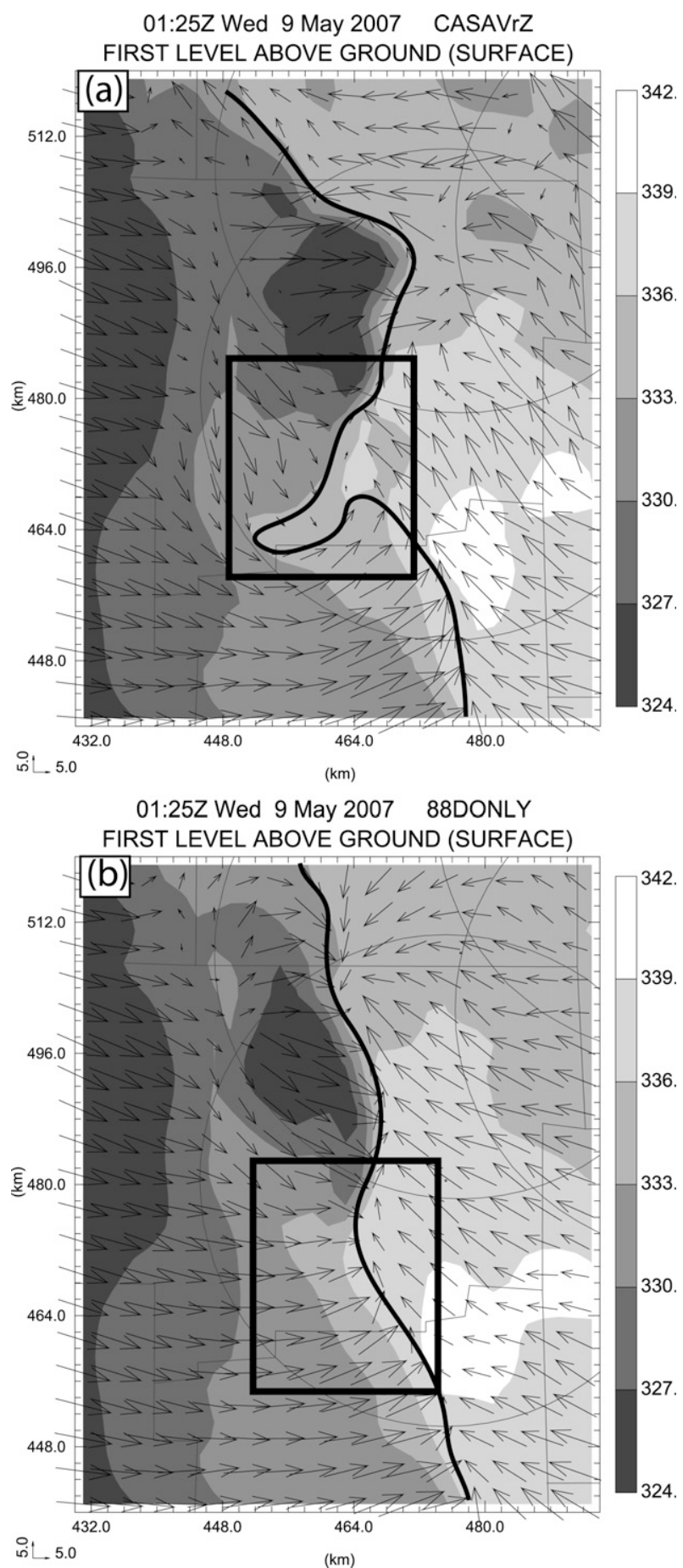

FIG. 6. Near-surface analyses of equivalent potential temperature (shaded in $3-\mathrm{K}$ intervals) and horizontal winds (vectors, $\mathrm{m} \mathrm{s}^{-1}$ ) at 0125 UTC 9 May 2007 from (a) CASAVrZ and (b) 88DONLY. The black square highlights an area of special interest where the two experiments are substantially different. The thick black line marks the approximate position of the gust front. surface wind and temperature observations from the Oklahoma Mesonet as these surface observations would mainly influence the analysis near the ground because of the specified short vertical decorrelation scale (see section 3).

The large impact of assimilating radar data is immediately apparent when comparing the 0200 UTC temperature and wind field analysis from 88DONLY to that of NORAD. In 88DONLY, the low-level cold pool associated with the MCS is well defined with a sharp gust front apparent along the leading edge of convection (Fig. 5b). This gust front structure is likely more accurate than that of NORAD, because radars can sample the gust front wind field with much higher resolution than the mesonet. We do note, however, that with the exception of the area close to the gust front the winds within, and to the east of, the cold pool are substantially weaker in 88DONLY than they are in NORAD. Comparison with Oklahoma Mesonet observations (see Figs. 5a,b) shows that the stronger winds in NORAD are more accurate than the weak winds in 88DONLY. The area of weak winds in $88 \mathrm{DONLY}$ is coincident with the zero isodop from the base scan KTLX being directly above the region (not shown). This suggests the 3DVAR analysis was unable to accurately estimate the cross-beam wind component in the vicinity of the zero isodop. Thus, while both NORAD and 88DONLY assimilate Mesonet data, the high-density zero-Vr measurements from KTLX cause the analyzed surface winds to be weak in this region of the domain in 88DONLY.

Farther aloft, we see significantly more structure in the wind field in 88DONLY than was present in NORAD (Figs. 4c vs 4b). For example, at grid level 7 (about $1.1 \mathrm{~km}$ AGL, denoted hereafter as $k=7$ ), there is strong southwesterly rear inflow behind the southern portion of the MCS, with easterly and northeasterly winds in the northern portion of the MCS indicating the presence of a broad LEV within the MCS. Radial velocity observations from KTLX and KFDR at 0200 UTC (see Fig. 1b) suggest that a cyclonic LEV was present within the convective line in the lower and midlevels of the atmosphere. The structure of this LEV is much better represented in 88DONLY than in NORAD. This is expected as WSR-88D data provides high-resolution $\mathrm{Vr}$ observations that are capable of resolving such features over a substantial vertical depth. In absence of radar data, the only wind observations with a significant vertical extent come from the wind profiler and raob networks, which have inadequate spatial resolutions to capture a LEV.

The differences between 88DONLY and CASAVrZ are present mainly near the surface and are less substantial than those between 88DONLY and NORAD. 


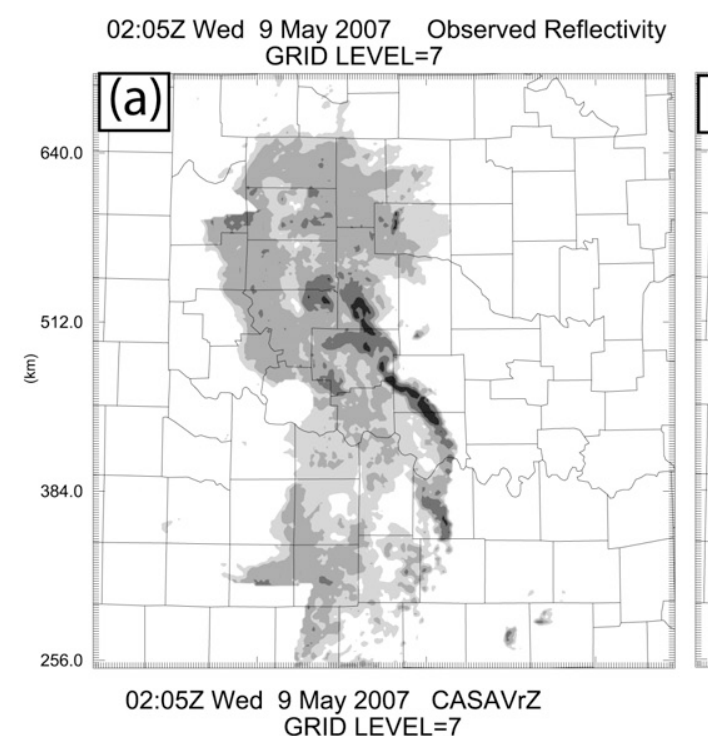

02:20Z Wed 9 May 2007 Observed Reflectivity
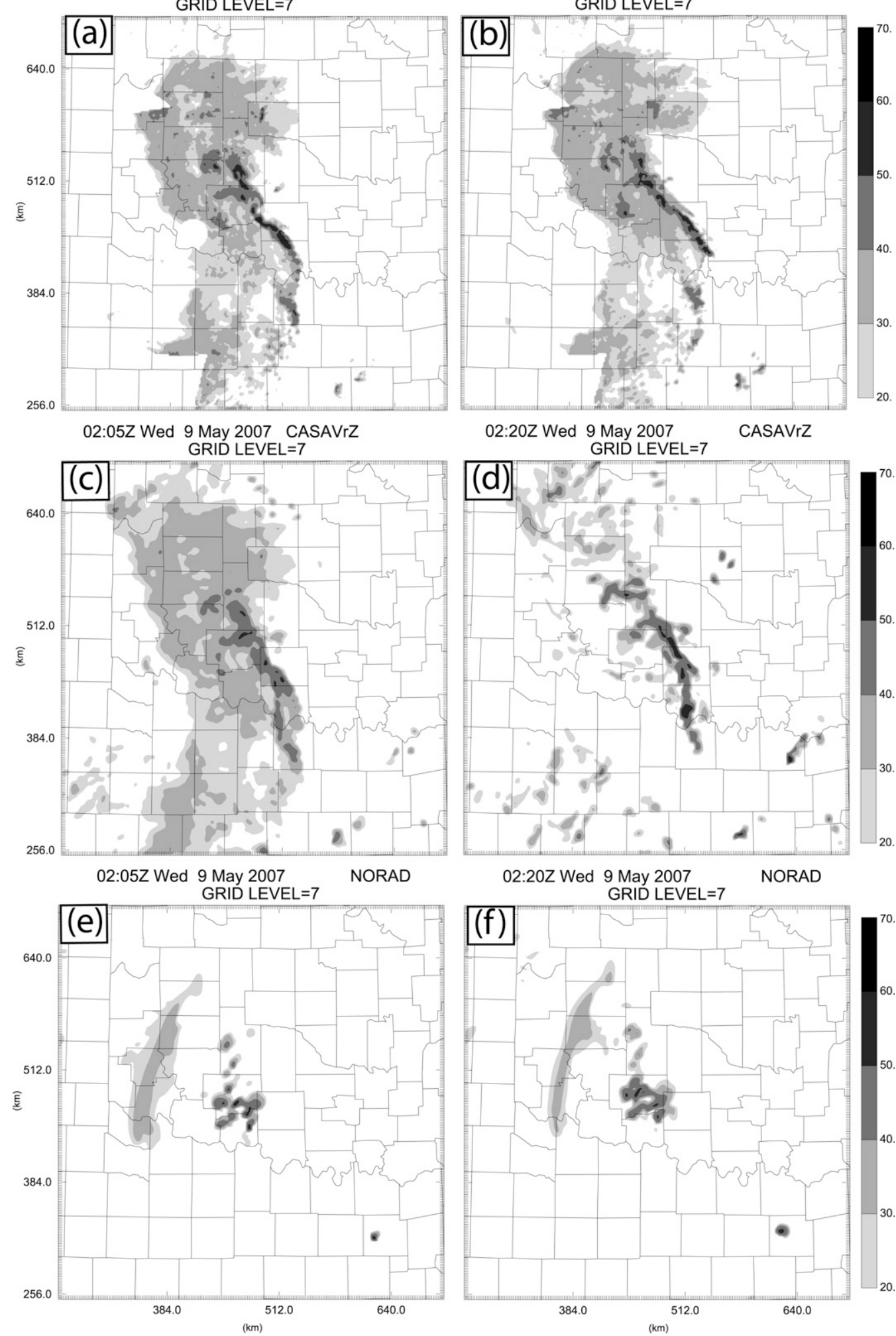

FIG. 7. Radar reflectivity factor (shaded, dBZ) at (top) grid level 7 observed by a mosaic of WSR-88D radars and forecast from (middle) CASAVrZ and (bottom) NORAD at (left) 0205 and (right) 0220 UTC 9 May 2007. 

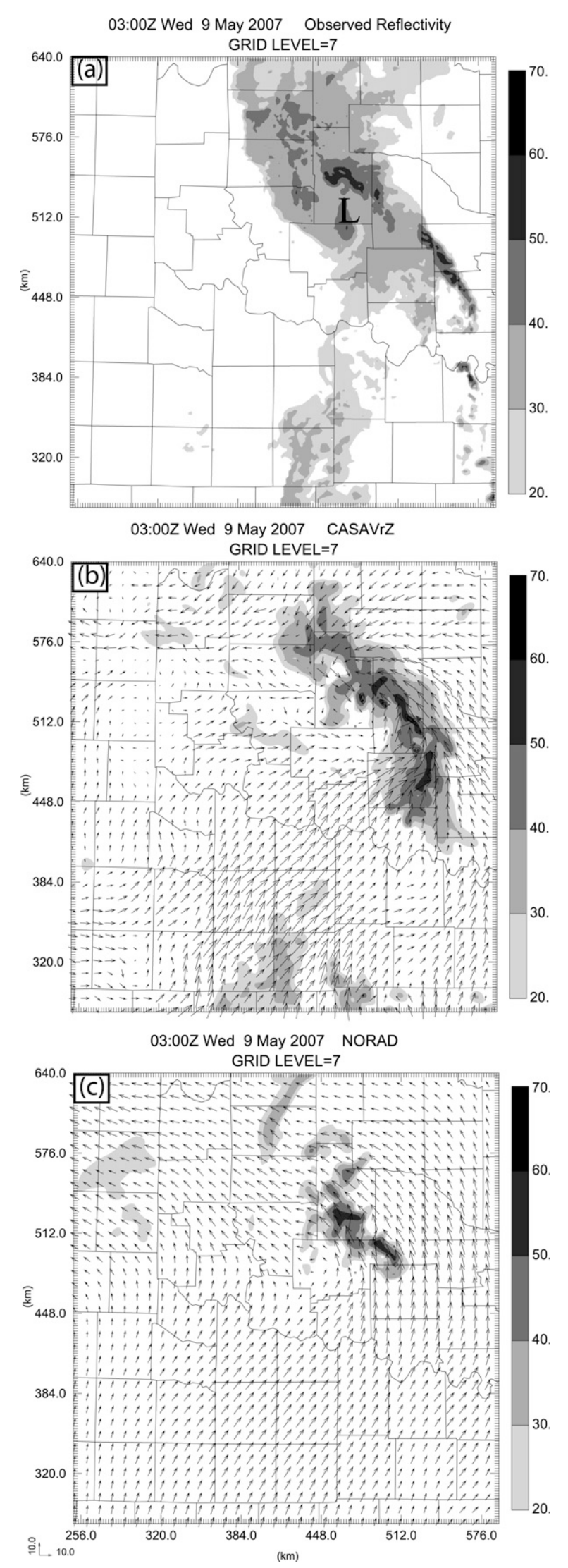

The area of weak winds that was present in 88DONLY behind, and in advance of, the gust front is not present in CASAVrZ (Fig. 5c). This occurs because low-level wind observations from the CASA radars, which indicate stronger northwest flow in this area, are closer to the surface and thus weighted more heavily in the assimilation procedure than base-level WSR-88D data whose influence comes from farther above the ground. More importantly, the gust front in CASAVrZ has more finescale structure than that of 88DONLY. In CASAVrZ, the gust front is wavy, with a surge of the cold pool in northern Comanche County. To the south of this surge, the gust front is about $15 \mathrm{~km}$ farther to the west. Immediately south of this buckle in the gust front there is another gust front surge into far western Stephens and Jefferson counties. In contrast, the gust front in 88DONLY is nearly linear, extending from southern Caddo County south-southeast into Jefferson County. The gust front structure in CASAZ (Fig. 5e) is similar to that of 88DONLY suggesting the wavy gust front structure in CASAVrZ is due to assimilating CASA Vr data. This is supported by the fact that the gust front orientation in CASAVr (Fig. 5d) is nearly identical to that of CASAVrZ. An examination of earlier times within the assimilation window of experiment CASAVr shows that assimilating CASA Vr data leads to variable wind speed and direction behind the gust front (Fig. 6a). This results in different portions of the gust front to move east at varying speeds during the assimilation window, thus leading to the wavy structure at 0200 UTC. This is likely due to the IP-1 network's ability to sample the lowlevel flow structure. 88DONLY and CASAZ have more uniform winds behind the gust front (Fig. 6b), leading to a more constant propagation speed for the entire gust front.

Overall, these results suggest that the assimilation of the CASA $Z$ data has little impact on the analysis whereas assimilating CASA Vr data leads to substantial differences in the analysis of the gust front position and orientation. Although this result seemingly contradicts previous studies that found a significant impact of reflectivity data assimilation (e.g., Hu et al. 2006a,b), the results can be reconciled. Hu et al. $(2006 \mathrm{a}, \mathrm{b})$ showed

FIG. 8. Observed reflectivity field (shaded, dBZ) from (a) the radar mosaic and forecast reflectivity (shaded, dBZ), horizontal wind vectors $\left(\mathrm{m} \mathrm{s}^{-1}\right)$, and vertical vorticity (contours at intervals of $100 \times 10^{-5} \mathrm{~s}^{-1}$ starting at $300 \times 10^{-5} \mathrm{~s}^{-1}$ ) from (b) CASAVrZ and (c) NORAD. All fields plotted at grid level 7 at 0300 UTC 9 May 2007. The "L" in (a) marks the approximate observed LEV location. 

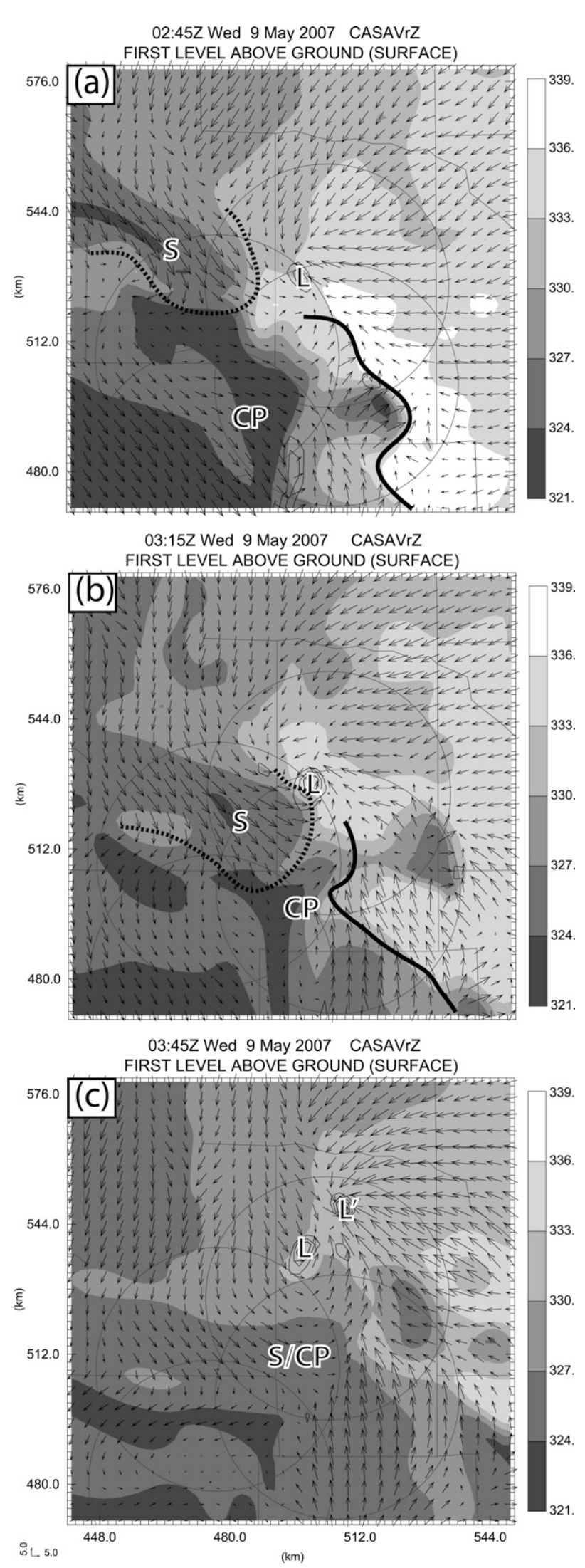

that assimilation of reflectivity has a large impact when compared to experiments that only assimilated conventional observations (i.e., from all platforms except radars). Our study finds a similar result when comparing NORAD and 88DONLY. However, we also find that assimilating CASA reflectivity data in addition to WSR-88D data has little impact because the influence of the WSR-88D reflectivity data through the cloud analysis can be felt at the low levels through the fall of hydrometeors and their impact on evaporation and subsequent cold pool development. Conversely, midlevel winds may differ significantly compared to lowlevel winds, thus the low-level wind generally cannot be inferred from midlevel wind observations. WSR-88Ds cannot observe the lowest $1 \mathrm{~km}$ of the atmosphere at the given distance (see Fig. 3a), whereas, the CASA IP-1 network observes the low levels of the current system. Thus, the assimilation of CASA Vr data has a substantial impact on the low-level wind analysis. It should be noted, however, that there is no independent observational platform with adequate spatial resolution to verify whether the linear gust front of 88DONLY (and CASAZ) or the wavy gust front of CASAVrZ (and CASAVr) is more accurate. Additionally, the impact of CASA Vr data is found only near the surface over a relatively small area (the CASA domain). Outside of this area, the RA experiments, not surprisingly, have few differences during the assimilation period.

\section{b. Forecast results}

We now examine the forecast period (0200-0500 UTC) from the experiments described in section 3a. Our focus is on the accuracy of LEV forecast, as well as whether the differences in the 0200 UTC analyses described above lead to substantial differences in the forecast period. As during the assimilation period, many features during the forecast period are very similar among the RA experiments. Accordingly, we describe the general evolution of the forecast period from all RA experiments while highlighting differences between them and NORAD as well as differences among the RA experiments.

FIG. 9. Forecast near-surface equivalent potential temperature (shaded, K) and horizontal wind vectors $\left(\mathrm{m} \mathrm{s}^{-1}\right)$ from CASAVrZ at (a) 0245, (b) 0315, and (c) 0345 UTC 9 May 2007; S corresponds to the secondary outflow surge, CP corresponds to the squall line generated cold pool, L represents the location of the center of the original LEV, while $\mathrm{L}^{\prime}$ corresponds to the new LEV center. In (a) and (b), the dashed and solid lines mark the approximate location of the secondary outflow surge and main convective cold pool gust fronts, respectively. 
Between 0200 and 0300 UTC, the large MCS present in the RA experiments across much of western Oklahoma and north Texas moves to the northeast. Figure 7 shows the observed reflectivity from the four WSR-88D mosaic (see section $4 \mathrm{~b}$ ) and forecast reflectivity from CASAVrZ and NORAD at 0200 and 0220 UTC. Most of the stratiform precipitation region associated with the MCS vanishes during this 20-min period of the RA experiments (Figs. 7c,d). Hu et al. (2006a,b) noted a similar adjustment period in the simulations presented in their work. They explain that the adjustment occurs in the model as the cloud variables adjust to better fit the model dynamics and physics.

This adjustment period is not necessary in NORAD because it does not use the cloud analysis procedure to adjust the cloud variables. As a result, the forecast from NORAD evolves with more continuity than that of the RA experiments (Figs. 7e,f). Nevertheless, the lack of assimilated radar data precludes a MCS in NORAD.

Figure 8 shows the observed reflectivity from the radar mosaic and forecast reflectivity from CASAVrZ and NORAD at 0300 UTC. By 0300 UTC, most of the convection in the RA experiments within Texas has dissipated while the LEV in the northern portions of the convection in southwest Oklahoma has intensified (Fig. 8b). A strong but narrow northwesterly secondary outflow surge is moving southeast into eastern Caddo County. A broad area of rotation exists between the secondary outflow surge and southeast flow within and in advance of the squall line. This rotation is most pronounced in CASAVrZ and CASAVr.

The evolution of the MCS in the RA experiments is similar to the behavior of the observed MCS, as reflectivity observations show that much of the precipitation in Texas dissipated by 0245 UTC (See Fig. 1c). This dissipation trend was already underway during the assimilation window, which shows the assimilation scheme correctly analyzed this trend and the model continued it in forecast period. Additionally, the vertical vorticity field is well forecast as radial velocity observations from KTLX indicate a broad LEV centered over Caddo County at 0300 UTC (see Fig. 8a). Convection becomes slightly more organized in NORAD by 0300 UTC (Fig. 8c), but its development is still significantly underforecast when compared to observations and the RA experiments.

The general evolution of the LEV in the RA experiments remains similar through the remainder of the forecast period. In all RA experiments, the LEV intensifies (i.e., it becomes more organized with higher maximum vorticity values) as it becomes collocated with a northwesterly secondary outflow surge (Fig. 9a). At the same time, stronger southeast flow and enhanced surface convergence develops to the northeast of the intensifying

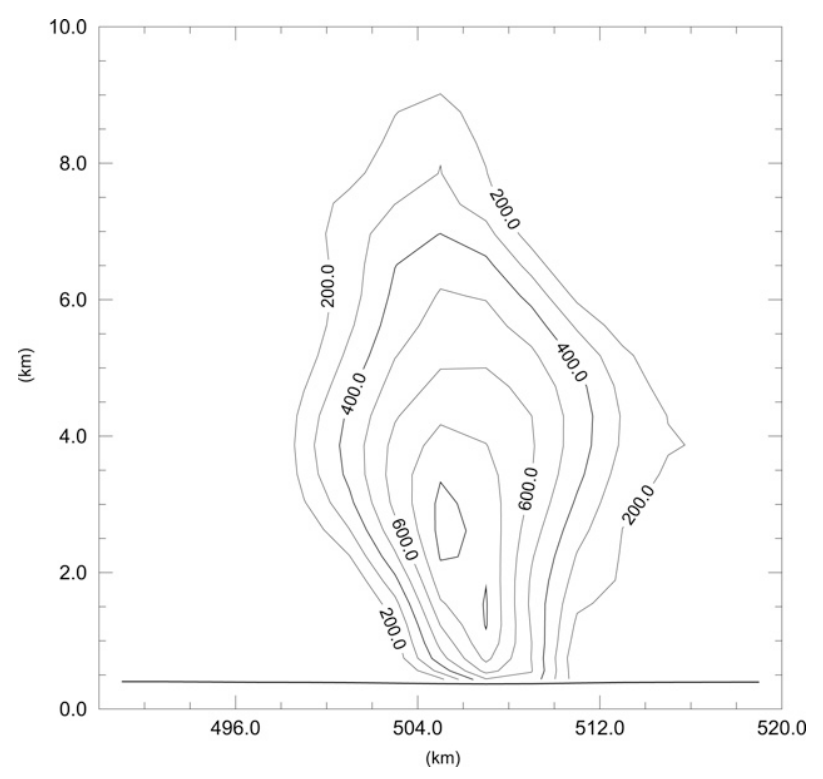

FIG. 10. An $X-Z$ cross section through the forecasted LEV in CASAVrZ at 0350 UTC 9 May 2007. Vertical vorticity is contoured in black at an interval of $100 \times 10^{-5} \mathrm{~s}^{-1}$ starting at $200 \times$ $10^{-5} \mathrm{~s}^{-1}$.

LEV (Fig. 9b). As this occurs, a strong convective cell forms to the northeast of the initial LEV center. The initial LEV center then decays as a new stronger center of rotation rapidly develops in the strong convection about $10 \mathrm{~km}$ northeast of the original LEV center (Fig. 9c). Low-level thermodynamic and wind observations of the 9 May LEV are too coarse to ascertain whether this redevelopment process actually occurred. However, as we noted in section 2, WSR-88D and Oklahoma Mesonet observations showed the LEV did rapidly intensify between 0245 and 0345 UTC. Additionally, like the observed LEV, the modeled LEV extends to the surface (Fig. 10). The evolution of the surface features in NORAD is less complex than that of the RA experiments and consists of the cold pool moving to the east at about $5 \mathrm{~m} \mathrm{~s}^{-1}$. Additionally, there is no indication of the LEV at the surface in NORAD.

Figures 11-13 compare the evolution of the observed MCS and LEV with the forecasted MCS and LEV in NORAD, 88DONLY, and CASAVrZ between 0350 and 0450 UTC. The evolution of CASAZ and CASAVr is not shown because they are nearly identical to 88DONLY and CASAVrZ, respectively. During this period, the LEV in the RA experiments moves northnortheast and continues to strengthen. As this occurs, the MCS develops into a comma-shaped echo that closely resembles both the observed reflectivity and the comma echo stage of the schematic presented in Fujita (1978). The LEV is embedded in the center of the convection composing the comma head. 

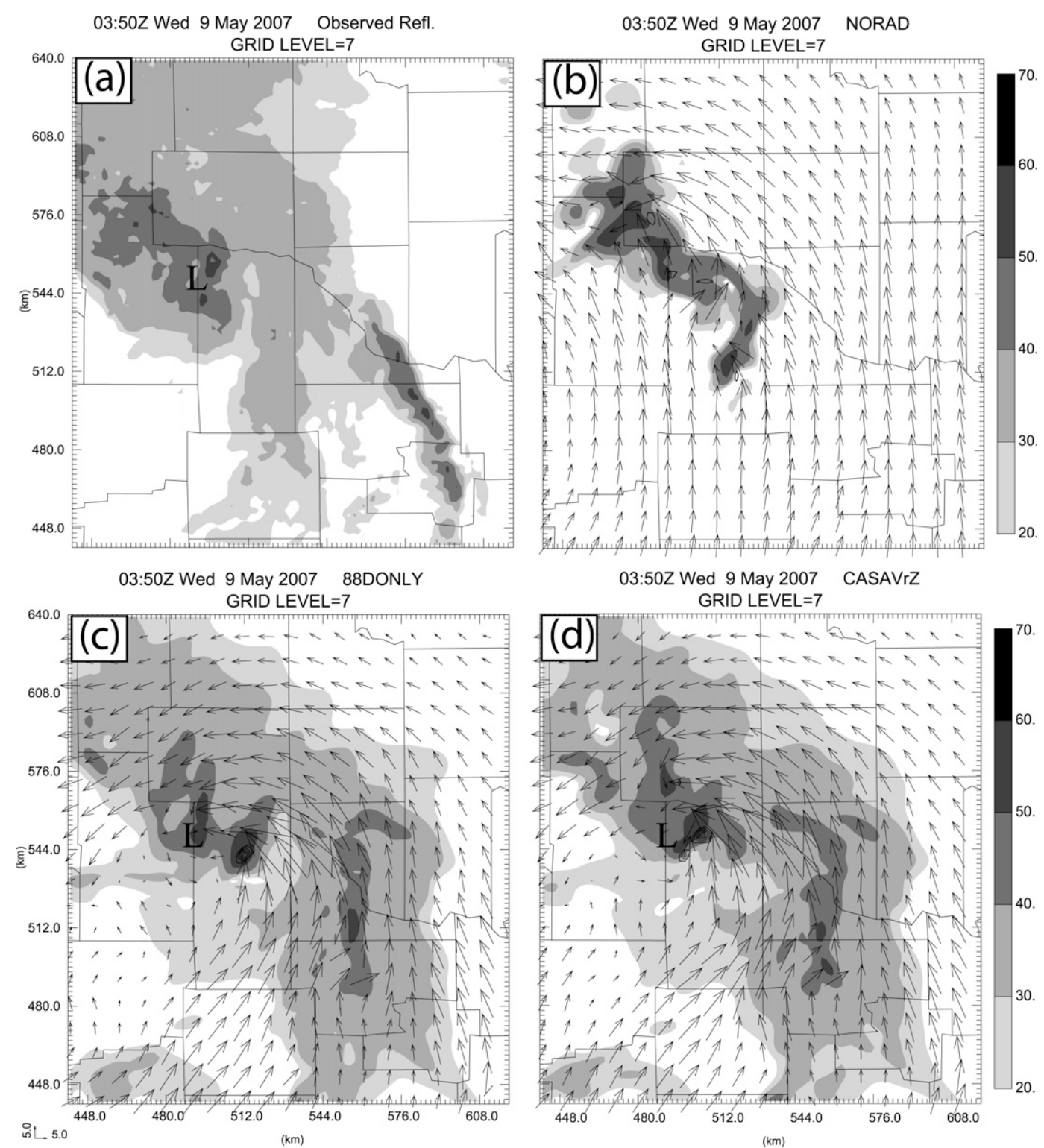

FIG. 11. The 0350 UTC 9 May 2007 (a) observed reflectivity (shaded, dBZ) and forecast reflectivity (shaded, $\mathrm{dBZ}$ ), horizontal wind vectors $\left(\mathrm{m} \mathrm{s}^{-1}\right.$ ), and vertical vorticity (contoured as in Fig. 8) from experiment (b) NORAD, (c) 88DONLY, and (d) CASAVrZ. The "L" in (a), (c), and (d) marks the approximate observed LEV location.

Comparison of NORAD with the RA experiments shows the RA experiments are much more accurate than NORAD. Like the RA experiments, NORAD develops a broad vortex that tracks through southwest and central Oklahoma. However, unlike the RA experiments that have a strong LEV and reflectivity pattern that closely resembles observations, the vortex in NORAD is weak and the reflectivity pattern is dissimilar to observations (Figs. 11b, 12b, and 13b).

While the general features of the RA experiments are similar, some differences do exist between them in the forecast development and location of the LEV. In 88DONLY and CASAZ, the LEV intensification and subsequent redevelopment occurs about $10 \mathrm{~km}$ farther south and east than that in CASAVrZ or CASAVr. Because of this displacement, the LEV in 88DONLY (Figs. 11c, 12c, and 13c) and CASAZ tracks about $10 \mathrm{~km}$ farther southeast than the LEV in CASAVrZ (Figs. 11d, 12d, and 13d) and CASAVr. KTLX Vr observations can be used to subjectively determine the LEV location. These observations show that the observed LEV tracked to the west of all of the RA experiments (see Figs. 11-13). 

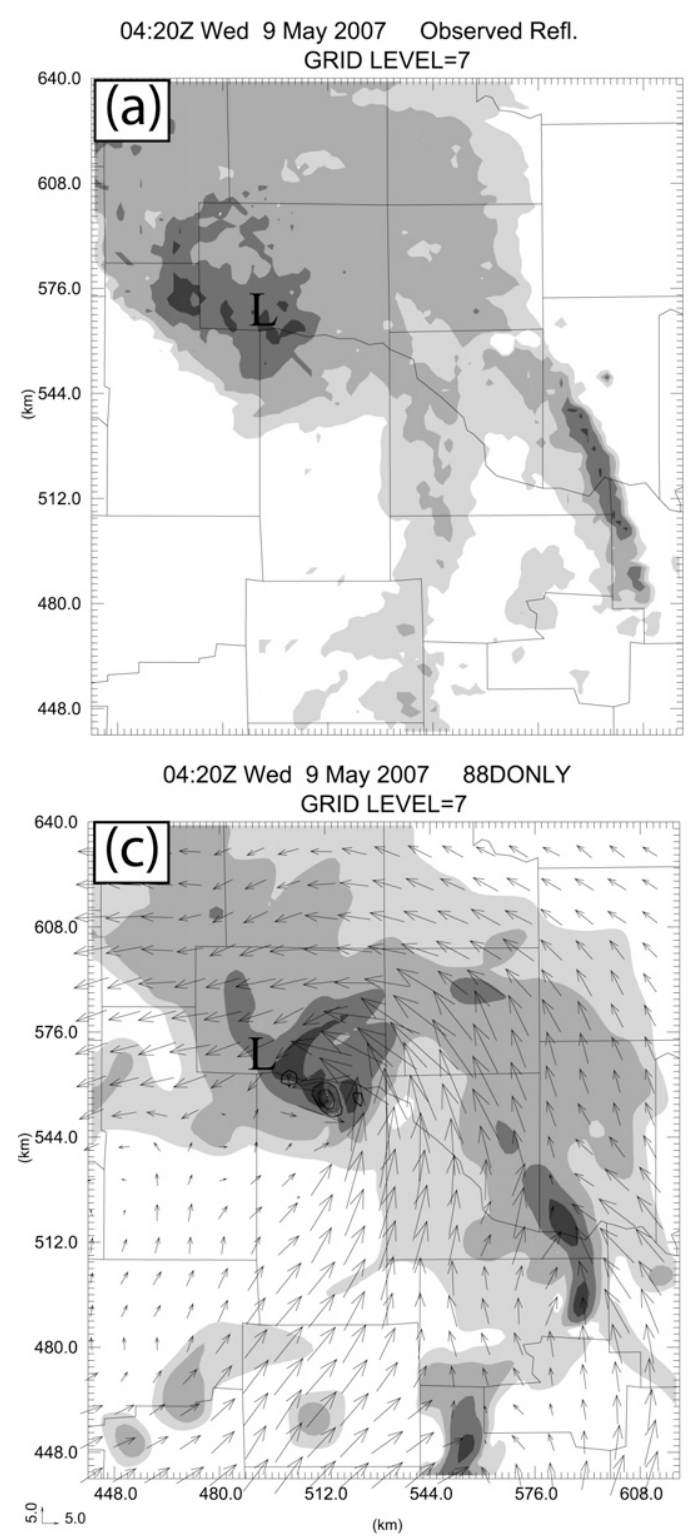
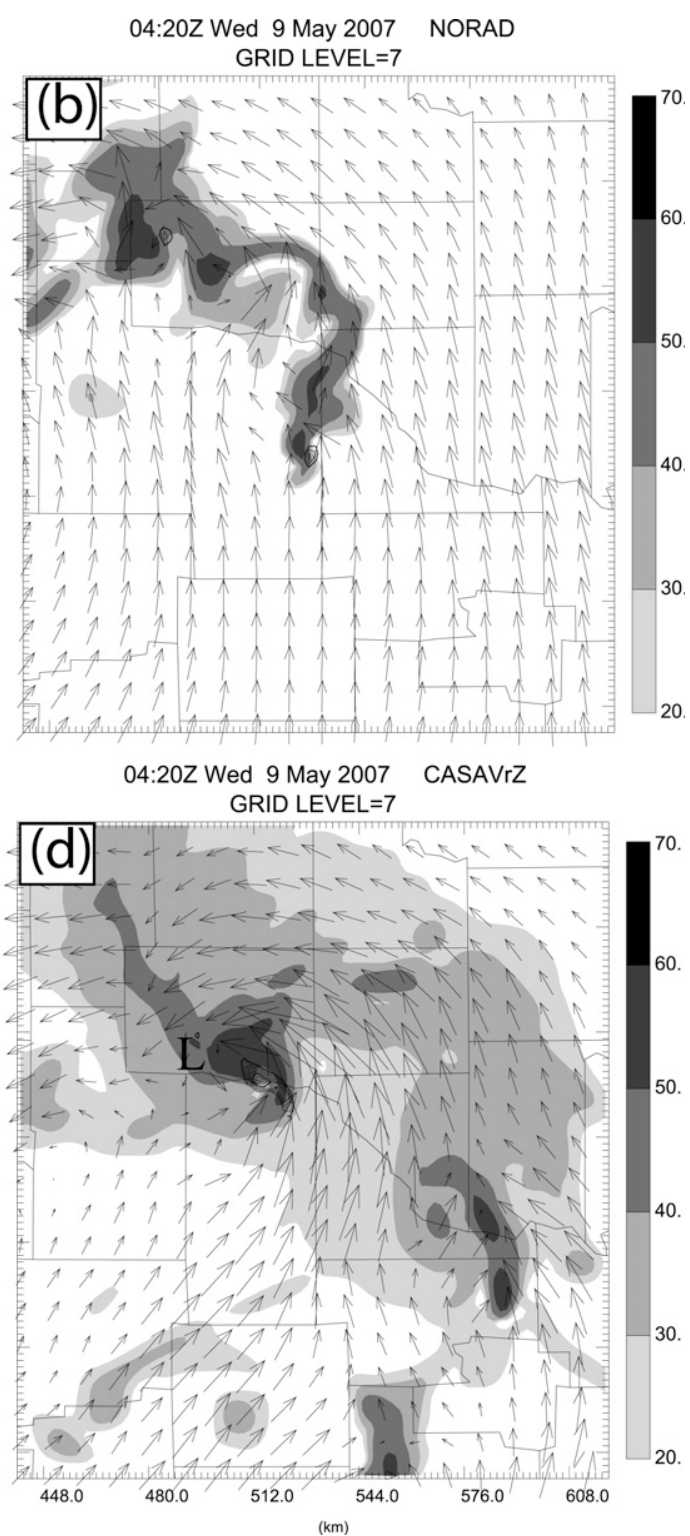

FIG. 12. As in Fig. 11, but at 0420 UTC 9 May 2007.

Thus, the position of the LEV in CASAVrZ and CASAVr is closer to the observed position than in the 88DONLY or CASAZ experiments.

To get a better qualitative sense of the accuracy of the forecast wind field, we can compare $\mathrm{Vr}$ observations from KTLX with simulated KTLX Vr data from CASAVrZ and NORAD (Fig. 14). This comparison shows that the LEV from CASAVrZ closely resembles the observed LEV with a large vortex signature present to the west-northwest of KTLX in both observed and simulated Vr. A broad area of low Vr values to the northeast and southeast of the forecast LEV indicate a larger cross-beam component to the flow than was observed, which suggests the forecast LEV has too large of a spatial extent in CASAVrZ. A similar pattern is seen in the simulated Vr signature of the LEV from all other RA experiments. In contrast, simulated Vr from NORAD indicates a very weak LEV that only vaguely resembles the observed $\mathrm{Vr}$ pattern.

The main reason for the differences in vortex intensity and location among the RA experiments appears to be related to the proximity of the squall line associated with the MCS to the northwesterly secondary outflow surge. Figures $15 \mathrm{a}-\mathrm{c}$ shows the evolution of the secondary outflow surge and LEV redevelopment in 88DONLY, which can be compared to that in CASAVrZ (originally presented in Fig. 9 and reproduced in Figs. 15d-f). At 0315 UTC in CASAVrZ and CASAVr, the gust front 

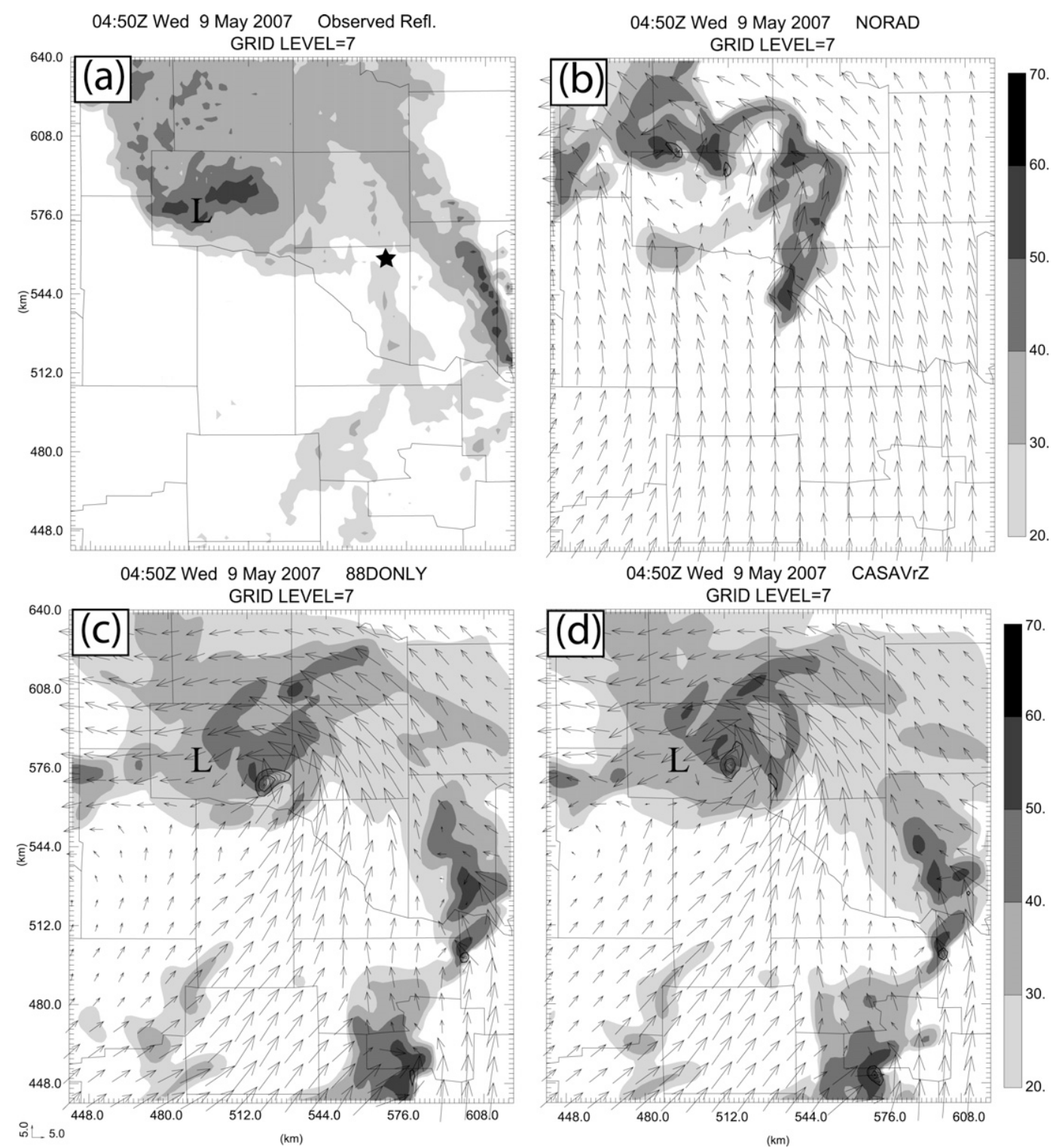

FIG. 13. As in Fig. 11, but at 0450 UTC 9 May 2007. The black star in (a) marks the location of the WSR-88D KTLX.

associated with the squall line is positioned $20-30 \mathrm{~km}$ farther west and north than it is in 88DONLY and CASAZ. Thus, as the secondary outflow surge moves southeast, it merges with the cold pool associated the squall line earlier in CASAVrZ and CASAVr than it does in 88DONLY and CASAZ. As the cold pools merge, the LEV intensifies. Thus, because the cold pools merge earlier and farther west in CASAVrZ and CASAVr, the LEV in these experiments develops and tracks farther west than it does in 88DONLY and CASAZ.

The underlying cause of the differences in the squallline structure and position is complex, but appears to be related to assimilating radial velocity data from CASA radars. In the assimilation period, we noted that main gust front in CASAVrZ and CASAVr had a structure that was substantially different than that of 88DONLY and CASAZ. While it is difficult to show concretely (due to the highly nonlinear nature of the problem), we speculate that the differences in the gust front location and structure in the during the assimilation period led to differences in the LEV and squall-line evolution. Thus, it seems likely that the capability of the CASA network to resolve low-level inflow during the assimilation yielded a more detailed squall-line structure, which resulted in 


\section{KTLX observed $\mathrm{Vr} \sim 0450$ UTC 9 May 2007}

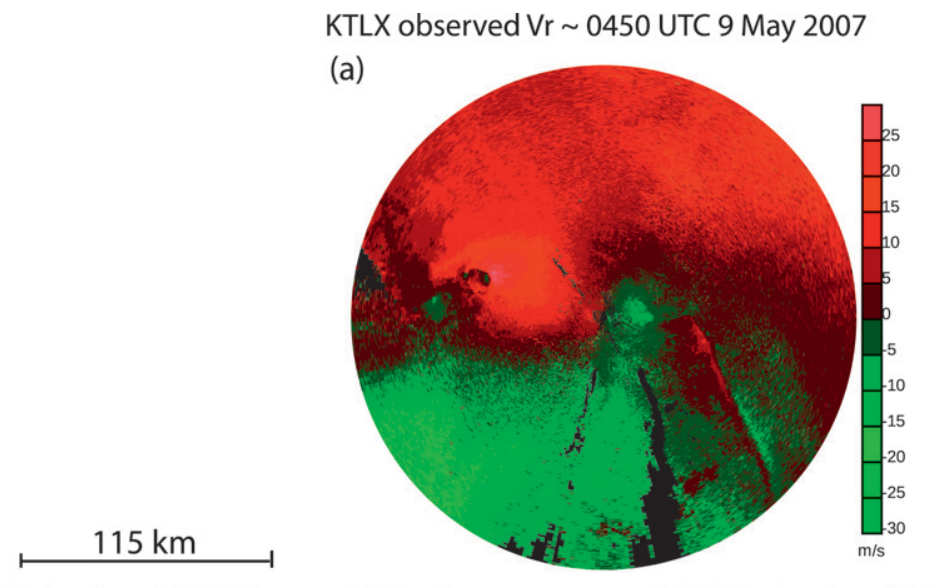

CASAVrZ simulated KTLX Vr 0450 UTC 9 May 2007

(b)

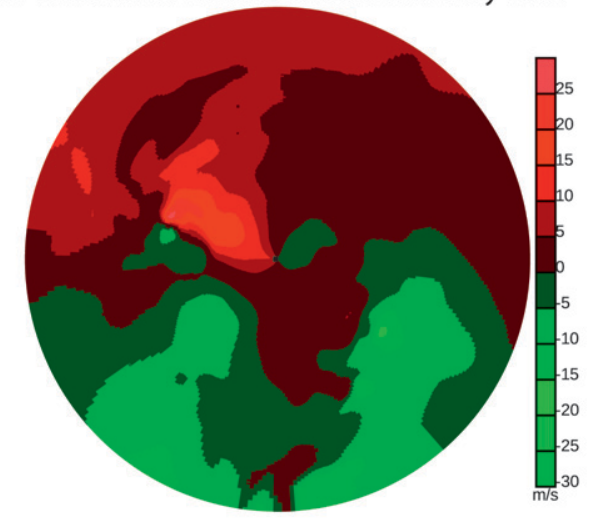

NORAD simulated KTLXVr 0450 UTC 9 May 2007

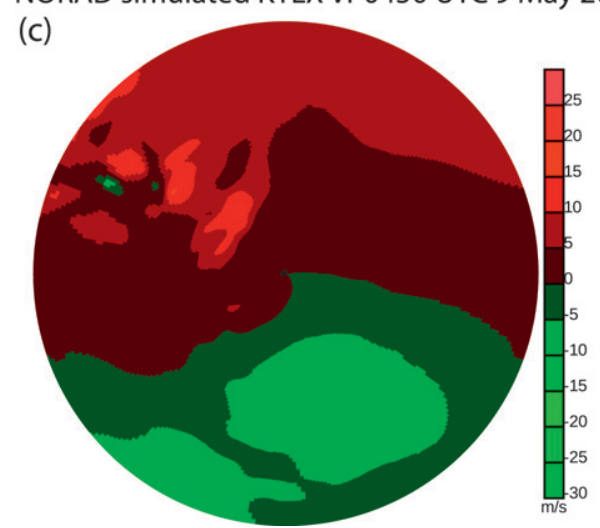

FIG. 14. Radial velocity (shaded in $\mathrm{m} \mathrm{s}^{-1}$ ) at 0450 UTC 9 May 2007 (a) observed from KTLX and (b) simulated from experiment CASAVrZ and (c) NORAD. Black data points in (a) are locations where data are missing. The radar is located at the center of plotted circles. The center of the observed LEV in (a) is located about $70 \mathrm{~km}$ west-northwest of KTLX.

a forecasted LEV development location and track that more closely resemble observations in experiments that assimilated CASA Vr data.

We can objectively quantify the differences in reflectivity pattern using $r_{c}$ and $N_{R}$ as described in section 4 . Calculations of $r_{c}$ for the forecast period are presented in Fig. 16a. The rapid decline in $r_{c}$ from 0.75 to 0.32 for the RA experiments between 0200 and 0235 UTC is the result of the dissipation of stratiform precipitation due to the model adjustment explained earlier. Thereafter, the $r_{c}$ increases sharply for the RA experiments and is around 0.55 from 0300 UTC onward. The $r_{c}$ for NORAD is substantially lower remaining around 0.45 for the same period. The $r_{c}$ for CASAVrZ and CASAVr are equal to or higher than those of 88DONLY and CASAZ throughout the entire forecast period. This is likely a reflection of the more accurate forecast of the LEV in CASAVrZ and CASAVr.

The evolution of forecast and observed $N_{R}$ is presented in Fig. 16b. After a brief adjustment period for the RA experiments from 0200 to 0220 UTC, we note remarkable correspondence between the RA-forecasted $N_{R}$ and the observed $N_{R}$ until 0400 UTC. All RA experiments indicate that $N_{R}$ gradually rises until it peaks around 0310 UTC, it then drops rapidly until 0400 UTC and levels off thereafter. This is very similar to the evolution of observed $N_{R}$ indicating that the RA experiments are fairly accurate with their forecast convective evolution. CASAVrZ and CASAVr capture the timing of the observed peak of $N_{R}$ at 0310 UTC and begin to level off closer to the observed time than 88DONLY and CASAZ. In contrast to the RA experiments, NORAD drastically underestimates $N_{R}$ until 0400 UTC and does not forecast the observed evolution; it instead indicates a gradual increase in $N_{R}$ throughout the forecast period. After 0400 UTC, the $N_{R}$ of NORAD is closer to the observed value because those of the RA experiments are too high due to spurious convection to the south and west of the LEV (see Figs. 12c,d and 13c,d).

Additional quantitative verification against $\mathrm{Vr}$ observations from KTLX was attempted. However, verification against KTLX Vr observations showed large errors 

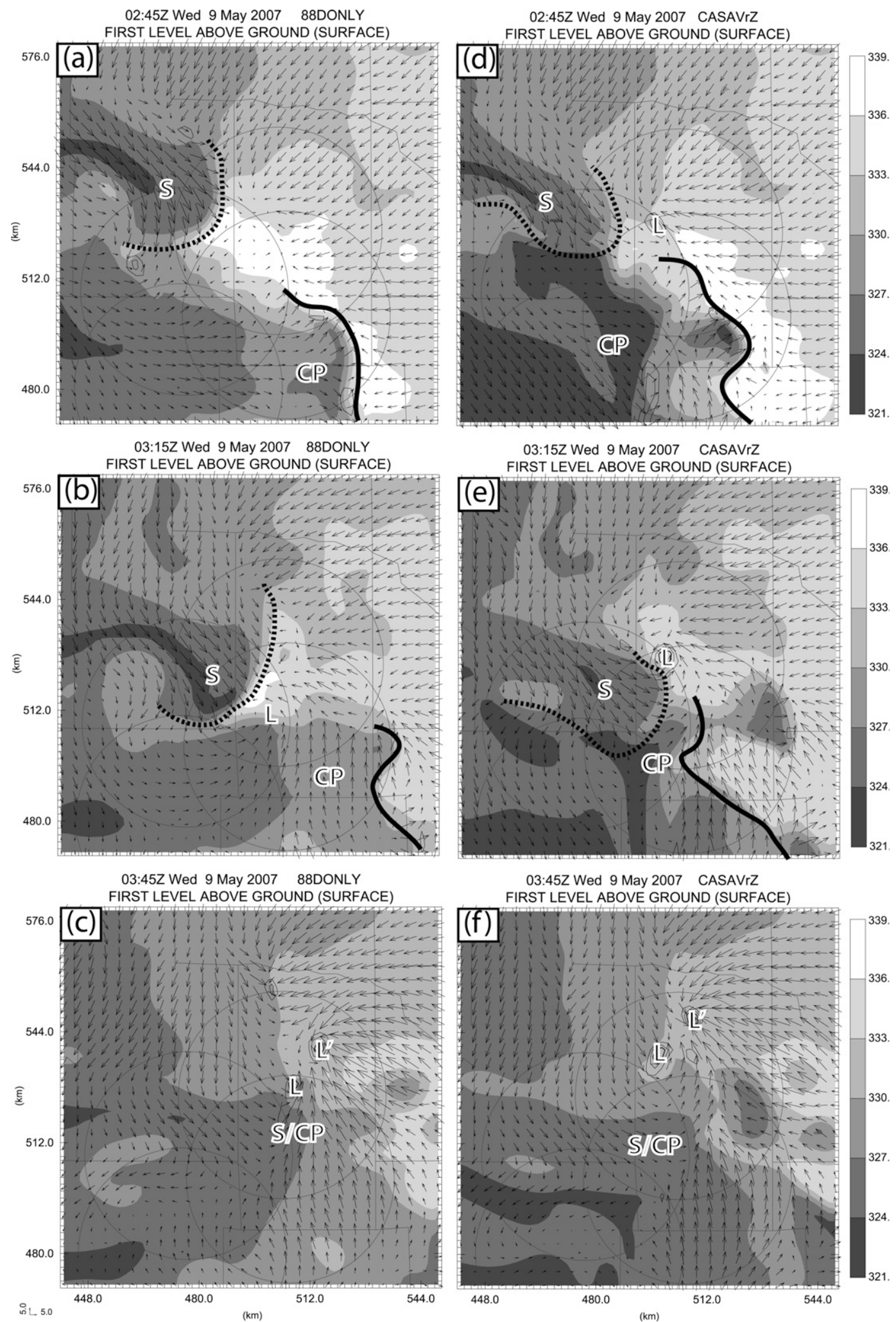

FIG. 15. (a)-(c) As in Fig. 9, but for 88DONLY and (d)-(f) reproduction of Fig. 9 for comparison purposes. 

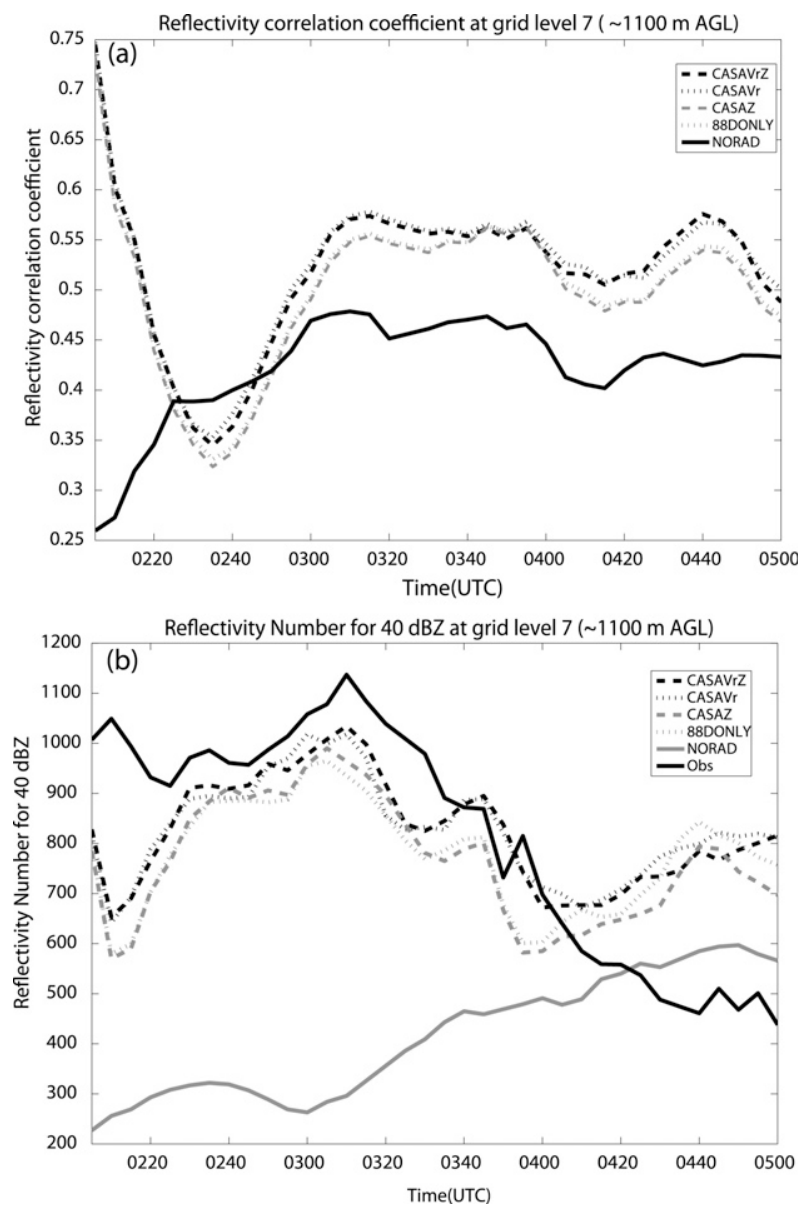

FIG. 16. (a) Reflectivity correlation coefficient calculated for the $15-\mathrm{dB} Z$ threshold and (b) number of reflectivity. Both statistics are calculated for all five experiments within the verification domain shown in Fig. 3a over the 0200-0500 UTC 9 May 2007 forecast period.

associated with error in the strength, size, and exact location of the forecast LEV. Because of these forecast errors, in particular those in size and position, the $\mathrm{Vr}$ difference field between forecast and observations for the RA experiments did not show smaller errors than that of NORAD, leading to comparable levels of pointwise root-mean-square errors for all experiments. However, we stress that qualitatively the forecast LEV $\mathrm{Vr}$ signature in the RA experiments more closely resembled the observed signature than in NORAD. This issue of disagreement between measures-based quantitative verification and subjective verification has been discussed and addressed in several recent studies (e.g., Davis et al. 2006a,b; Marzban and Sandgathe 2006; Ebert 2009). Verification was also attempted against CASA-IP1 $\mathrm{Vr}$ observations, but was ineffective because the LEV and MCS moved out of the CASA domain around 0330 UTC.
Overall, our quantitative verification using reflectivity agrees well with our qualitative comparison of the forecast fields. Both verification methods indicate that a substantially better forecast was produced by the RA experiments than that of NORAD. Additionally, like the qualitative evaluation, quantitative verification against reflectivity indicates a slight improvement from the assimilation of CASA Vr data.

\section{Discussion and conclusions}

In this study, we describe results from a set of $2-\mathrm{km}$ resolution experiments of a cyclonic LEV that developed within an MCS on 9 May 2007 in southwest and central Oklahoma. Intermittent data assimilation cycles are performed using the ARPS 3DVAR. Experiments are designed to examine the impact of assimilating radar data from WSR-88D as well as determine if any additional benefit is obtained from assimilating CASA data.

Results show that the experiments that assimilate radar data produce forecasts of the 9 May 2007 LEV that qualitatively evolve in a way closely resembling the observed evolution of the LEV. Qualitative and quantitative comparison between observed and model forecast reflectivity fields reveals good correspondence between observed and modeled features.

In addition to revealing good accuracy, examination of the analyses and forecasts from the experiments shows a small but important impact from assimilating CASA data (in addition to the assimilation of WSR-88D data). In particular, assimilating CASA Vr data led to differences in the analysis of the gust front structure and location. These differences led to a more accurate forecast evolution of the MCS and LEV in experiments that used CASA Vr data.

The size of the CASA domain relative to the computational domain in the present study was small and thus the impacts from CASA were only realized over a small fraction of the grid. This problem is expected to be alleviated with the planned expansion of the IP-1 network to have at least twice as many radars (McLaughlin et al. 2009). Furthermore, the 2-km grid spacing is fairly coarse compared to the $100-\mathrm{m}$ gate spacing of CASA radars. In a future study, we plan on increasing the model resolution by several times. In doing so we hope to more fully explore the potential of high-resolution CASA data to impact the analysis and prediction of the substorm-scale rotational and convective structures, including observed mesovortices, in this case.

Acknowledgments. This work was primarily supported by NSF Grant EEC-0313747, as part of ERC CASA. Partial support was also provided by Grants ATM0530814, ATM-0738370, and ATM-0802888. Ming Xue 
was also supported by NSF Grants ATM-0608168, ATM-0750790, ATM-0941491, and OCI-0905040, and by Grant 40620120437 from the Chinese Natural Science Foundation. Thanks to Matt Kumjian for comments on this paper. Harold Brooks and Altuğ Aksoy are thanked for consultation on verification techniques. Jerald Brotzge is thanked for facilitating the use of CASA data. Comments from three anonymous reviewers also helped strengthen the content of this manuscript. Oklahoma Mesonet data were provided courtesy of the Oklahoma Mesonet, a cooperative venture between Oklahoma State University and The University of Oklahoma and supported by the taxpayers of Oklahoma. The computing for this project was performed at the OU Supercomputing Center for Education and Research (OSCER) at the University of Oklahoma (OU).

\section{REFERENCES}

Aksoy, A., D. C. Dowell, and C. Snyder, 2010: A multicase comparative assessment of the ensemble Kalman filter for assimilation of radar observations. Part II: Short-range ensemble forecasts. Mon. Wea. Rev., 138, 1273-1292.

Brewster, K., 1996: Application of a Bratseth analysis scheme including Doppler radar data. Preprints, 15th Conf. on Weather Analysis and Forecasting, Norfolk, VA, Amer. Meteor. Soc., 92-95.

_- 2002: Recent advances in diabatic initialization of a non-hydrostatic numerical model. Preprints, 19th Conf. on Weather Analysis and Forecasting/15th Conf. on Numerical Weather Prediction, San Antonio, TX, Amer. Meteor. Soc., J6.3. [Available online at http://ams.confex.com/ams/SLS_WAF_NWP/ techprogram/paper_47414.htm.]

_- M. Hu, M. Xue, and J. Gao, 2005: Efficient assimilation of radar data at high resolution for short-range numerical weather prediction. Preprints, WWRP Int. Symp. on Nowcasting and Very Short Range Forecasting, Toulouse, France, WWRP, 3.06.

— , K. W. Thomas, J. Brotzge, Y. Wang, D. Weber, and M. Xue, 2007: High resolution data assimilation of CASA X-band radar data for thunderstorm forecasting. Preprints, 22nd Conf. on Weather Analysis and Forecasting/18th Conf. on Numerical Weather Prediction, Salt Lake City, UT, Amer. Meteor. Soc., 1B.1. [Available online at http://ams.confex.com/ams/22WAF18NWP/ techprogram/paper_124619.htm.]

Brock, F. V., K. C. Crawford, R. L. Elliott, G. W. Cuperus, S. J. Stadler, H. L. Johnson, and M. D. Eilts, 1995: The Oklahoma Mesonet: A technical overview. J. Atmos. Oceanic Technol., 12, 5-19.

Brotzge, J., K. Hondl, B. Phillips, L. Lemon, E. J. Bass, D. Rude, and D. L. Andra Jr., 2010: Evaluation of distributed collaborative adaptive sensing for the detection of low-level circulation and implications for severe weather warning operations. Wea. Forecasting, 25, 173-189.

Caya, A., J. Sun, and C. Snyder, 2005: A comparison between the 4D-VAR and the ensemble Kalman filter techniques for radar data assimilation. Mon. Wea. Rev., 133, 3081-3094.

Chen, F., and J. Dudhia, 2001: Coupling an advanced land surfacehydrology model with the Penn State-NCAR MM5 modeling system. Part I: Model implementation and sensitivity. Mon. Wea. Rev., 129, 569-585.

Davis, C., B. Brown, and R. Bullock, 2006a: Object-based verification of precipitation forecasts. Part I: Methodology and application to mesoscale rain areas. Mon. Wea. Rev., 134, 1772-1784.

,$- \ldots$, and $—, 2006 \mathrm{~b}$ : Object-based verification of precipitation forecasts. Part II: Application to convective rain systems. Mon. Wea. Rev., 134, 1785-1795.

Dawson, D. T., II, and M. Xue, 2006: Numerical forecasts of the 1516 June 2002 Southern Plains severe MCS: Impact of mesoscale data and cloud analysis. Mon. Wea. Rev., 134, 1607-1629.

Ebert, E. E., 2009: Neighborhood verification: A strategy for rewarding close forecasts. Wea. Forecasting, 24, 1498-1510.

Evensen, G., 1994: Sequential data assimilation with a nonlinear quasi-geostrophic model using Monte Carlo methods to forecast error statistics. J. Geophys. Res., 99, 10 143-10 162.

Fujita, T. T., 1978: Manual of downburst identification for project Nimrod. University of Chicago SMRP Research Paper 156, $104 \mathrm{pp}$.

Gao, J., M. Xue, K. Brewster, and K. K. Droegemeier, 2004: A three-dimensional variational data analysis method with recursive filter for Doppler radars. J. Atmos. Oceanic Technol., 21, 457-469.

Houze, R. A., Jr., S. A. Rutledge, M. I. Biggerstaff, and B. F. Smull, 1989: Interpretation of Doppler weather radar displays of midlatitude mesoscale convective systems. Bull. Amer. Meteor. Soc., 70, 607-619.

$\mathrm{Hu}, \mathrm{M}$., and M. Xue, 2007: Impact of configurations of rapid intermittent assimilation of WSR-88D radar data for the 8 May 2003 Oklahoma City tornadic thunderstorm case. Mon. Wea. Rev., 135, 507-525.

$\longrightarrow$, — and K. Brewster, 2006a: 3DVAR and cloud analysis with WSR-88D level-II data for the prediction of Fort Worth tornadic thunderstorms. Part I: Cloud analysis and its impact. Mon. Wea. Rev., 134, 675-698.

,$- \ldots$, J. Gao, and K. Brewster, 2006b: 3DVAR and cloud analysis with WSR-88D level-II data for the prediction of Fort Worth tornadic thunderstorms. Part II: Impact of radial velocity analysis via 3DVAR. Mon. Wea. Rev., 134, 699-721.

Kessler, E. I., 1969: On the Distribution and Continuity of Water Substance in Atmospheric Circulations. Meteor. Monogr., No. 32, Amer. Meteor. Soc., 84 pp.

Kong, F., and Coauthors, 2009: A real-time storm-scale ensemble forecast system: 2009 spring experiment. Preprints, 23rd Conf. on Weather Analaysis and Forecasting/19th Conf. on Numerical Weather Prediction, Omaha, NE, Amer. Meteor. Soc., 16A.3. [Available online at http://ams.confex.com/ams/23WAF19NWP/ techprogram/paper_154118.htm.]

Lin, Y.-L., R. D. Farley, and H. D. Orville, 1983: Bulk parameterization of the snow field in a cloud model. J. Climate Appl. Meteor., 22, 1065-1092.

Marzban, C., and S. Sandgathe, 2006: Cluster analysis for verification of precipitation fields. Wea. Forecasting, 21, 824-838.

McLaughlin, D., and Coauthors, 2009: Short-wavelength technology and the potential for distributed networks of small radar systems. Bull. Amer. Meteor. Soc., 90, 1797-1817.

Rogers, R. R., and M. K. Yau, 1989: A Short Course in Cloud Physics. 3rd ed. Pergamon Press, 293 pp.

Schaefer, J. T., 1990: The critical success index as an indicator of warning skill. Wea. Forecasting, 5, 570-575.

Snook, N., and M. Xue, 2008: Effects of microphysical drop size distribution on tornadogenesis in supercell thunderstorms. Geophys. Res. Lett., 35, L24803, doi:10.1029/2008GL035866. 
Snyder, C., and F. Zhang, 2003: Assimilation of simulated Doppler radar observations with an ensemble Kalman filter. Mon. Wea. Rev., 131, 1663-1677.

Stensrud, D. J., and Coauthors, 2009: Convective-scale warn-onforecast system. Bull. Amer. Meteor. Soc., 90, 1487-1499.

Sun, J., 2005: Initialization and numerical forecasting of a supercell storm observed during STEPS. Mon. Wea. Rev., 133, 793-813. , and N. A. Crook, 1997: Dynamical and microphysical retrieval from Doppler radar observations using a cloud model and its adjoint. Part I: Model development and simulated data experiments. J. Atmos. Sci., 54, 1642-1661.

— and _ 1998: Dynamical and microphysical retrieval from Doppler radar observations using a cloud model and its adjoint. Part II: Retrieval experiments of an observed Florida convective storm. J. Atmos. Sci., 55, 835-852.

— D. W. Flicker, and D. K. Lilly, 1991: Recovery of threedimensional wind and temperature fields from simulated singleDoppler radar data. J. Atmos. Sci., 48, 876-890.

Tong, M., and M. Xue, 2005: Ensemble Kalman filter assimilation of Doppler radar data with a compressible nonhydrostatic model: OSS experiments. Mon. Wea. Rev., 133, 1789-1807.

Weisman, M. L., 1993: The genesis of severe long-lived bow-echoes. J. Atmos. Sci., 50, 645-670.

— , and C. A. Davis, 1998: Mechanisms for the generation of mesoscale vortices within quasi-linear convective systems. J. Atmos. Sci., 55, 2603-2622.

Weygandt, S. S., A. Shapiro, and K. K. Droegemeier, 2002a: Retrieval of model initial fields from single-Doppler observations of a supercell thunderstorm. Part I: Single-Doppler velocity retrieval. Mon. Wea. Rev., 130, 433-453.

$\longrightarrow, \ldots$, and ——, 2002b: Retrieval of model initial fields from single-Doppler observations of a supercell thunderstorm. Part II: Thermodynamic retrieval and numerical prediction. Mon. Wea. Rev., 130, 454-476.

Wolf, P. L., 1998: WSR-88D radar depiction of supercell-bow echo interaction: Unexpected evolution of a large, tornadic, "comma shaped" supercell over eastern Oklahoma. Wea. Forecasting, 13, 492-504.

Xiao, Y., M. Xue, W. J. Martin, and J. Gao, 2005: Development of an adjoint for a complex atmospheric model, the ARPS, using TAF. Automatic Differentiation: Applications, Theory, and Implementations, H. M. Bücker et al., Eds., Springer, 263-272.
Xue, M., and W. J. Martin, 2006: A high-resolution modeling study of the 24 May 2002 case during IHOP. Part I: Numerical simulation and general evolution of the dryline and convection. Mon. Wea. Rev., 134, 149-171.

— K. K. Droegemeier, and V. Wong, 2000: The Advanced Regional Prediction System (ARPS) - A multiscale nonhydrostatic atmospheric simulation and prediction tool. Part I: Model dynamics and verification. Meteor. Atmos. Phys., 75, 161-193.

— System (ARPS) - A multiscale nonhydrostatic atmospheric simulation and prediction tool. Part II: Model physics and applications. Meteor. Atmos. Phys., 76, 143-165.

—, D.-H. Wang, J.-D. Gao, K. Brewster, and K. K. Droegemeier, 2003: The Advanced Regional Prediction System (ARPS), storm-scale numerical weather prediction and data assimilation. Meteor. Atmos. Phys., 82, 139-170.

_- M. Tong, and K. K. Droegemeier, 2006: An OSSE framework based on the ensemble square-root Kalman filter for evaluating impact of data from radar networks on thunderstorm analysis and forecast. J. Atmos. Oceanic Technol., 23, 46-66.

— , and Coauthors, 2009: CAPS real-time multi-model convectionallowing ensemble and 1-km convection-resolving forecasts for the NOAA Hazardous Weather Testbed 2009 Spring Experiment. Preprints, 23rd Conf. on Weather Analysis and Forecasting/19th Conf. on Numeral Weather Prediction, Omaha, NE, Amer. Meteor. Soc., 16A.2. [Available online at http://ams. confex.com/ams/23WAF19NWP/techprogram/paper_154323. htm.]

Zhang, F., C. Snyder, and J. Sun, 2004: Impacts of initial estimate and observations on the convective-scale data assimilation with an ensemble Kalman filter. Mon. Wea. Rev., 132, 1238-1253.

Zhang, J., 1999: Moisture and Diabatic Initialization Based on Radar and Satellite Observation. School of Meteorology, University of Oklahoma, $194 \mathrm{pp}$.

_ , F. Carr, and K. Brewster, 1998: ADAS cloud analysis. Preprints, 12th Conf. on Numerical Weather Prediction, Phoenix, AZ, Amer. Meteor. Soc., 185-188.

Zhao, K., and M. Xue, 2009: Assimilation of coastal Doppler radar data with the ARPS 3DVAR and cloud analysis for the prediction of Hurricane Ike (2008). Geophys. Res. Lett., 36, L12803, doi:10.1029/2009GL038658. 